\title{
De-ubiquitination of SAMHD1 by USP7 overcomes oncogenic stress and contributes to chemotherapy insensitivity
}

Jingwei Liu

the First Affiliated Hospital of China Medical University, and Key Laboratory of Cancer Etiology and

Prevention (China Medical University),

Tingting Zhou

China Medical University

xiang dong

Institute of Translational Medicine

Qiqiang Guo

Institute of Translational Medicine

Lixia Zheng

Institute of Translational Medicine

Xiaoxun Wang

Institute of Translational Medicine

Naijin Zhang

China Medical University

Li Danni

the First Hospital of China Medical University

Ling Ren

Institute of Translational Medicine

Fei Yi

China Medical University

Ying Zhang

China Medical University

Zi-wei Li

China Medical University

Xi-wen Wang

China Medical University

chengsi deng

Institute of Translational Medicine

Chunlu Li

Institute of Translational Medicine 


\section{Hongde Xu}

China Medical University

\section{Yi Guan}

China Medical University

\section{Xiaoman Li}

China Medical University

\section{Yang Yu}

China Medical University

\section{Wendong Guo}

China Medical University

\section{Zhuo Wang}

China Medical University

\section{Bo Jiang}

China Medical University

\section{Xuan Wu}

China Medical University

\section{Ning Bai}

China Medical University

\section{Yanling Feng}

Institute of Translational Medicine

\section{Mengtao Ma}

Institute of Translational Medicine

\section{Qingquan Kong}

Institute of Translational Medicine

\section{Jiayi Wei}

Institute of Translational Medicine

\section{Zhenshuang Wang}

Department of Anus and Intestine Surgery, First Affiliated Hospital of China Medical University

\section{Hao Li}

the First Affiliated Hospital of China Medical University, and Key Laboratory of Cancer Etiology and

Prevention (China Medical University),

\section{Xiaoyu Song}

China Medical University https://orcid.org/0000-0002-6606-327X

\section{Chengzhong Xing}

the First Affiliated Hospital of China Medical University, and Key Laboratory of Cancer Etiology and Prevention (China Medical University), https://orcid.org/0000-0001-6463-019X

\section{Liu Cao ( Icao@cmu.edu.cn )}

China Medical University https://orcid.org/0000-0001-6471-1993 
Article

Keywords: USP7, SAMHD1, ubiquitination, cancer

Posted Date: August 20th, 2021

DOl: https://doi.org/10.21203/rs.3.rs-763082/v1

License: (c) (i) This work is licensed under a Creative Commons Attribution 4.0 International License. Read Full License 


\section{De-ubiquitination of SAMHD1 by USP7 overcomes oncogenic stress and contributes to chemotherapy insensitivity}

Jingwei Liu ${ }^{1,2}$, Tingting Zhou ${ }^{1,2}$, Xiang Dong ${ }^{1,2}$, Qiqiang Guo ${ }^{1,2}$, Lixia Zheng ${ }^{1,2}$, Xiaoxun Wang ${ }^{1,2}$, Naijin Zhang ${ }^{1,2}$, Danni $\mathrm{Li}^{1,2}$, Ling Ren ${ }^{1,2}$, Fei Yi ${ }^{1,2}$, Ying Zhang ${ }^{1,2}$, Ziwei $\mathrm{Li}^{1,2}$, Xiwen Wang ${ }^{1,2}$, Chengsi Deng ${ }^{1,2}$, Chunlu $\mathrm{Li}^{1,2}$, Hongde $\mathrm{Xu}^{1,2}$, Yi Guan ${ }^{1,2}$, Xiaoman $\mathrm{Li}^{1,2}$, Yang $\mathrm{Yu}^{1,2}$, Wendong Guo ${ }^{1,2}$, Zhuo Wang ${ }^{1,2}$, Bo Jiang ${ }^{1,2}$, Xuan $\mathrm{Wu}^{1,2}$,

Ning Bai ${ }^{1,2}$, Yanling Feng ${ }^{1,2}$, Mengtao $\mathrm{Ma}^{1,2}$, Qingquan Kong ${ }^{1,2}$, Jiayi $\mathrm{Wei}^{4}$, Zhenshuang Wang ${ }^{3}, \mathrm{Hao} \mathrm{Li}^{5}$, Xiaoyu Song ${ }^{1,2 *}$, Chengzhong Xing ${ }^{3 *}$, Liu Cao ${ }^{1,2 *}$.

1. The College of Basic Medical Science, China Medical University, Shenyang, Liaoning Province, China

2. Key Laboratory of Cell Biology of Ministry of Public Health, and Key Laboratory of Medical Cell Biology of Ministry of Education, Liaoning Province Collaborative Innovation Center of Aging Related Disease Diagnosis and Treatment and Prevention, China Medical University, Shenyang, Liaoning Province, China

3. Department of Anus and Intestine Surgery, First Affiliated Hospital of China Medical University, Shenyang, Liaoning Province, China

4. Department of Developmental Cell Biology, Key Laboratory of Cell Biology, Ministry of Public Health, and Key Laboratory of Medical Cell Biology, Ministry of Education, China Medical University, Shenyang, Liaoning Province, China.

5. Department of Laboratory Medicine, The First Affiliated Hospital of China Medical University, Shenyang, Liaoning Province, China.

*Correspondence: Liu Cao, 1cao@cmu.edu.cn; Chengzhong Xing, xcz1966@126.com; Xiaoyu Song, xysong@cmu.edu.cn. 


\begin{abstract}
Oncogenic stress induces DNA damage response (DDR) that guards against genetic instability during the evolution of cancer. SAMHD1, a dNTPase protecting cells from viral infections, has been recently found to participate in DNA damage repair process. However, its role in tumorigenesis remains largely unknown. Here, we show that SAMHD1 is up-regulated in early-stage human carcinoma tissues and cell lines under oxidative stress or genotoxic insults. We further demonstrate that de-ubiquitinating enzyme USP7 interacts with SAMHD1 and de-ubiquitinates it at lysine 421, thus stabilizing SAMHD1 protein expression, and promotes tumor cell survival under genotoxic stress. Furthermore, SAMHD1 levels positively correlates with USP7 in various human carcinomas, and is associated with an unfavorable survival outcome in patients who underwent chemotherapy. Moreover, USP7 inhibitor sensitizes tumor cells to chemotherapeutic agents by decreasing SAMHD1 in vitro and in vivo. These findings suggest that targeting USP7 may help overcome chemoresistance, thus necessitating further investigation in the pursuit of precision medicine.
\end{abstract}

Key words: USP7, SAMHD1, ubiquitination, cancer.

Running title: USP7-SAMHD1 axis and cancer 


\section{Introduction}

Balance between DNA damage and DNA damage response (DDR) enable cells to maintain genome integrity against endogenous and exogenous insults ${ }^{1}$. An underlying hallmark of cancers is their genomic instability, which is characterized by a greater propensity to accumulate DNA damage ${ }^{2}$. As a result, cancer cells demonstrate increased genomic instability and a greater dependency on DDR pathways to overcome frequent DNA damage ${ }^{3}$. A variety of chemotherapeutics induce DNA damage to kill tumor cells. However, activated DDR in cancer cells can repair DNA damage induced by genotoxic insults thus leading to chemoresistance ${ }^{4}$. Therefore, understanding the key regulators and mechanisms orchestrating DDR may potentially elucidate mechanisms of tumorigenesis and provide novel therapeutic target for cancer patients 5 .

SAMHD1, an important dNTP hydrolase, maintains nucleic acid metabolism and genome stability by regulating the abundance of the dNTP pool in cells ${ }^{6,7}$. The enzyme was first found to be correlated with Aicardi-Goutières syndrome, suggesting that SAMHD1 participates in immune regulation and innate immunity ${ }^{8,9}$. In addition, SAMHD1 restricts DNA viruses and retroviruses by virtue of its dNTP hydrolysis ability, and this direct action on viral genetic material has a broad spectrum ${ }^{10}$. At present, most of the studies focused on the importance of antiviral role of SAMHD ${ }^{11}$, while the potential role of SAMHD1 in tumorigenesis and therapy remains largely elusive. Recently, SAMHD1 was found to promotes DNA end resection to facilitate DNA double-strand break (DSB) repair by interacting with CtIP independent of its dNTP hydrolysis ability ${ }^{12}$. DSBs are the most cytotoxic type of DNA damage, as unrepaired or inappropriate repair of DSBs inevitably causes mutations or chromosomal aberrations ${ }^{13}$. Considering its role in DSB repair, SAMHD1 might be implicated in tumorigenesis and response to chemotherapy in cancer.

Emerging evidence have indicated that multiple post-translational protein modifications including ubiquitination are actively involved in the DDR process ${ }^{14,15}$. Deubiquitinating enzyme (DUB) is responsible for removing the ubiquitination modification of the substrate, maintaining the stability of the substrate, and preventing 
it from degradation by the proteasome ${ }^{16}$. Ubiquitin-specific proteases (USPs) constitute the largest DUB subfamily, of which USP7 is a key deubiquitinating enzyme stabilizing multiple substrates through deubiquitination, thereby regulating a variety of cellular processes including immune response, virus replication and cancer $^{17,18,19,20}$. However, the mechanistic insights into the role of USP7 in tumorigenesis and DDR remain to be elucidated.

In this study, we have demonstrated that USP7 interacts with SAMHD1 at the HD domain and deubiquitinates SAMHD1 at K421, thus stabilizing SAMHD1 by reducing its degradation via proteasome pathway. USP7-stabilized SAMHD1 promotes DDR by interacting with DSB repair initiator CtIP, therefore repairing DNA damage induced by reactive oxygen species (ROS) or genotoxic insults. Attesting to the function, both USP7 and SAMHD1 proteins were highly expressed in human carcinomas of various organs and their expressions were positively correlated. Most importantly, the USP7 inhibitor sensitizes tumor cells to chemotherapy by decreasing SAMHD1, which implies the potential of targeting USP7-SAMHD1-CtIP axis to improve chemosensitivity.

\section{Results}

ROS induces deubiquitination and increase of SAMHD1 for DNA damage repair

A number of previous studies have indicated the implication of SAMHD1 in the initiation and development of cancer, while inconsistent findings have been obtained in different tumor types ${ }^{21}$. To explore the role of SAMHD1 in tumorigenesis, we first examined the SAMHD1 expression by immunohistochemistry in several early-stage (stages I and II) human cancers including colonic and lung adenocarcinomas as well as thyroid carcinoma. To that end, semiquantitative immunohistochemical analysis revealed that SAMHD1 was highly expressed in carcinomas when compared to their paired peritumor tissues, suggesting that SAMHD1 might be implicated in tumor progression induced, at least in part, by elevated DNA damage and overcome oncogenic stress (Fig. 1A-1F; Fig. S1A-1C).

The occurrence and development of tumors are accompanied by the up-regulation of ROS and the accumulation of DNA damage, and ROS can induce 
DNA damage and activate the DNA damage response (DDR) signaling 22,23 . We found that oxidative stress generated by $\mathrm{H}_{2} \mathrm{O}_{2}$ elevated SAMHD1 in both $\mathrm{H} 1299$ (Fig. 1G) and HCT116 cells (Fig. S2A), thus confirming the change of SAMHD1 in response to oxidative stress in tumor cells. In addition, N-acetylcysteine (NAC), a scavenger of free radicals, significantly inhibited the SAMHD1 increase induced by $\mathrm{H}_{2} \mathrm{O}_{2}$ (Fig. $1 \mathrm{H}$; Fig. S2B). Quantitative PCR analysis revealed unchanged mRNA levels of SAMHD1 upon ROS stimulation (Fig. S2C), suggesting that post-translational modifications rather than transcriptional regulation is responsible for SAMHD1 protein overexpression. Indeed, the ubiquitination of SAMHD1 was significantly decreased under the stimulation of ROS (Fig. 1I), thus further supporting the importance of deubiquitination in upregulating SAMHD1 protein under oxidative stress. Previous studies have suggested that SAMHD1 promotes DNA end resection to facilitate DNA double-strand break repair by interacting with CtIP. In current study, we have demonstrated that ROS promotes the interaction of SAMHD1 and CtIP (Fig. 1J). This is in keeping with the observations that $\mathrm{H}_{2} \mathrm{O}_{2}$ induces formation of SAMHD1 and CtIP foci as well as their co-localization with DNA damage marker $\gamma \mathrm{H} 2 \mathrm{AX}$, respectively (Fig. 1K, 1L).

\section{USP7 interacts with SAMHD1 at the HD domain and stabilizes its protein expression}

Although the ubiquitination modification of SAMHD1 has been widely reported, the deubiquitinating enzyme (DUB) of SAMHD1 is still unknown. We next performed mass spectroscopy to identify potential molecules interacting with SAMHD1 (Fig. 2A). The observations in this regard suggested that USP7 might be a specific DUB for SAMHD1, a notion supported by the subsequent co-immunoprecipitation assays in HCT116 (Fig. 2B, C), Hela (Fig. S3A, B) and H1299 (Fig. S3C, D) cells. The USP7-SAMDH1 interaction was further confirmed by the in vitro glutathione-S-transferase (GST)-pulldown assays (Fig. 2D, E). To identify the specific USP7 binding domain in SAMHD1, we constructed different GST-SAMHD1 fusion proteins lacking each domain for the subsequent GST-pulldown assays (Fig. 2F). To that end, GST-SAMHD1 lacking the HD domain 
failed to interact with USP7, suggesting that it is essential for USP7-SAMHD1 interaction (Fig. 2G). Furthermore, co-localization of USP7 and SAMHD1were observed by confocal microscopy in Hela (Fig. 2H) and HEK293 (Fig. S3E) cells, respectively.

DUB deubiquitinates substrate and maintains the stability of the substrate, preventing it from degradation by the proteasome ${ }^{24}$. We next examined the effect of USP7 on SAMHD1 stability by overexpressing USP7 in HCT116 cells. To that end, SAMHD1 protein levels were gradually increased with the increased amount of USP7 plasmids transfected (Fig. 3A). In contrast, all three different siRNAs for USP7 reduced SAMHD1 expression in HCT116 cells (Fig. 3B). P5091, a specific USP7 inhibitor, decreased SAMHD1 levels in both concentration and time dependent manners in HCT116 (Fig. 3C; Fig. S3G) and H1299 (Fig. S3F) cells, respectively. Proteasome inhibitor MG132 prolonged the half-life of SAMHD1 protein under CHX treatment, suggesting that ubiquitin-proteasome pathway is a key manner for SAMHD1 protein degradation (Fig. 3D, E). Overexpressing USP7 wildtype also lengthened the half-life of SAMHD1 protein, in contrast to the effect of USP7 C223S, an inactivation mutant of the deubiquitination enzyme (Fig. 3F, G). Conversely, stable knockdown of USP7 (Fig. 3H, I) or treatment with P5091 shortened the half-life of SAMHD1 (Fig. 3J, K).

\section{USP7 deubiquitinates SAMHD1 K48-linked polyubiquitination at K421}

To explore the effect of USP7 on SAMHD1 ubiquitination, we next overexpressed Flag-USP7. As a result, deubiquitination of SAMHD1 protein was found in both HCT116 (Fig. 4A) and HEK293 (Fig. S4A) cells, respectively. In contrast, stable knockdown of USP7 induced ubiquitination of SAMHD1 protein in HCT116 cells (Fig. 4B). Administration of P5091 significantly increased ubiquitination of SAMHD1 protein in HCT116 (Fig. 4C), SW480 (Fig. S4B), Hela (Fig. S4C) and HEK293 (Fig. S4D) cells, respectively. In addition, overexpressing USP7 deubiquitinating enzyme inactivation mutant plasmid C223S failed to deubiquitinate SAMHD1 (Fig. 4D).

We next sought to identify the specific deubiquitination sites of SAMHD1 
protein by USP7 via ubiquitination site mass spectrometry. The study to that end revealed K421, K492, K494 as potential ubiquitination sites (Fig. S4G-I). Co-immunoprecipitation assays showed decreased ubiquitination when lysine $(\mathrm{K})$ K421 or K492 of SAMHD1 was substituted by arginine (R) (Fig. S4E). Further experiments demonstrated that SAMHD1 K492R and K494R, but not K421R, could be deubiquitinated by USP7, indicating that K421 is the specific site for USP7 deubiquitinating SAMHD1 (Fig. 4E-G). K421 is evolutionarily conservative across different species (Fig. S4F). Lastly, we transfected different HA-Ub plasmids including WT, K0, K48 and K63 along with USP7 and SAMHD1. To that end, USP7 specifically removes K48-linked polyubiquitination of SAMHD1 (Fig. 4H).

\section{SAMHD1 promotes cell survival and reduces apoptosis under genotoxic insults}

It has been recently reported that SAMHD1 participates in DNA DSB repair. This has led us to explore the effect of SAMHD1 on tumor cell survival under genotoxic insults. Consequently, stable knockdown of SAMHD1 significantly reduces the survival rate of HCT116 and SW480 cells in a cisplatin and doxorubicin concentration-dependent manner, respectively (Fig. 5A, 5C; Fig. S5A). In contrast, stable overexpression Flag-SAMHD1 in shSAMHD1 colon cancer cells increases the survival rate in a cisplatin and doxorubicin concentration-dependent manner, respectively (Fig. 5B, D; Fig. S5B). Moreover, SAMHD1 promotes cell survival in a cisplatin treatment time-dependent manner under genotoxic insults in HCT116 and SW480 cells, respectively (Fig. S5C-S5F).

We next investigated the effect of SAMHD1 on the apoptosis of tumor cells induced by genotoxic insults. Under the treatment of cisplatin, shSAMHD1 cells demonstrated significantly increased apoptosis when compared to shNC cells, while stably expressing Flag-SAMHD1 cells significantly decreased apoptosis when compared to vector cells stably expressing Flag in HCT116 and SW480 cells, respectively (Fig. 5E, 5F; Fig. S5G, 5H). To elucidate whether the inhibitory effect of SAMHD1 on cell apoptosis depends on its ability of dNTP hydrolase, we stably expressed H206A/H207A SAMHD1 with no dNTP hydrolase activity in shSAMHD1 HCT116 and SW480 cells, respectively. Under the treatment of cisplatin, SAMHD1 
wild-type and SAMHD1 AA both suppressed cell apoptosis in HCT116 and SW480 cells, respectively, with no statistical difference between their inhibitory effects, suggesting that SAMHD1 inhibition of cell apoptosis does not depend on the dNTP hydrolase activity (Fig. 5G). This notion was further confirmed by western blot analysis, in which significant increased apoptotic markers cleaved-PARP and cleaved-Caspase 3, and DSB marker $\gamma \mathrm{H} 2 \mathrm{AX}$ were observed in shSAMDH1 cells under the treatment of cisplatin, when compared to shNC cells (Fig. 5I). In addition, stably expressing Flag-SAMHD1 in colon cancer cells significantly decreased cleaved-PARP, cleaved-Caspase 3 and $\gamma \mathrm{H} 2 \mathrm{AX}$ when compared to Flag vector (Fig. 5J). Moreover, cisplatin induced formation of SAMHD1 and CtIP foci and their co-localization with $\gamma \mathrm{H} 2 \mathrm{AX}$, respectively (Fig. 5H; Fig. S5I).

It is widely known that chemotherapeutics elevate intracellular ROS levels, which contributes to their genotoxicity ${ }^{25,26}$. We have thereby further verified that cisplatin and doxorubicin increase SAMHD1 protein expression without altering its mRNA level (Fig. S2D-G). Moreover, these agents induce the deubiquitination of SAMHD1 protein (Fig. S2H, S2I) and promote the interaction of SAMHD1 and CtIP (Fig. S2J, S2K).

\section{The USP7-SAMHD1 axis interactions facilitate cell survival and reduce apoptosis under genotoxic insults}

To further elucidate the regulation of USP7 on SAMHD1 protein stability, we next explored the effect of USP7-SAMHD1 axis on tumor cell survival under genotoxic insults. First, cisplatin was found to promote the binding of USP7 and SAMHD1 both endogenously and exogenously (Fig. 6A; Fig. S6A, S6B). Similar results were also observed with doxorubicin (Fig. S6C). The USP7 inhibitor P5091 suppresses the increase of SAMHD1 protein induced by cisplatin or doxorubicin (Fig. 6B; Fig. S6D). In addition, the formation of SAMHD1 foci and co-localization with DNA damage marker $\gamma \mathrm{H} 2 \mathrm{AX}$ induced by cisplatin was significantly inhibited by P5091 (Fig. 6C). Similar findings were obtained with respect to the downstream CtIP foci and co-localization with $\gamma \mathrm{H} 2 \mathrm{AX}$ (Fig. 6D), suggesting that USP7-SAMHD1-CtIP axis contributes to DDR induced by chemotherapeutic agents. 
Stable knockdown of USP7 significantly reduced the survival rate of HCT116 cells in a cisplatin and doxorubicin concentration-dependent manner (Fig. 6E; Fig. S6E). Conversely, stable overexpression of Flag-SAMHD1 in shUSP7 HCT116 cells increased the survival rate (Fig. 6F; Fig. S6F). As for cell apoptosis, shUSP7 cells demonstrated significantly increased apoptosis when compared to shNC cells under the treatment of cisplatin or doxorubicin, whereas stably expressing Flag-SAMHD1 cells in shUSP7 cells showed significantly decreased apoptosis when compared to the HCT116 cells stably expressing the Flag vector (Fig. 6G, 6H; Fig. S6G, S6H). P5091 treatment significantly increased apoptosis induced by cisplatin or doxorubicin (Fig. 6I; Fig. S6I). The results were further confirmed by western blot analysis, in which apoptotic markers cleaved-PARP and cleaved-Caspase 3 and DSB marker $\gamma \mathrm{H} 2 \mathrm{AX}$ were significantly increased in shUSP7 HCT116 cells when compared to shNC cells under treatment of cisplatin (Fig. 6J). In addition, stably expressing Flag-SAMHD1 in shUSP7 HCT116 cells significantly decreased cleaved-PARP, cleaved-Caspase 3 and $\gamma \mathrm{H} 2 \mathrm{AX}$ when compared to cells expressing Flag vector (Fig. 6K).

\section{Inhibition of the USP7-SAMHD1 axis improves chemosensitivity}

To further explore the role of USP7-SAMHD1 in human cancers, semiquantitative immunohistochemical analyses for USP7 and SAMHD1 were performed in tumorous tissues. To that end, significantly higher levels of USP7 were found in colonic and lung adenocarcinomas as well as thyroid carcinoma when compared to their paired peritumoral tissues (Fig. 7A-F; Fig. S7A-S7C). Moreover, a significant positive correlation between USP7 and SAMHD1 expression was identified in all three tumor types, suggesting that the existence of the USP7-SAMHD1 axis in various human cancers (Fig. 7G-I). Furthermore, data from The Cancer Genome Atlas (TCGA) also indicate a positive correlation between USP7 and SAMHD1 expressions in various cancer types (Fig. 7J, 7K; Fig. S7D-S7K). In addition, co-localization of USP7 and SAMHD1 was identified in colonic adenocarcinoma cells while this phenomenon was only rarely observed in peritumoral cells (Fig. 7L).

We then analyzed the potential prognostic significance of SAMHD1 expression in 
patients with various cancers who received chemotherapy according to TCGA data. To that end, a higher SAMHD1 expression is significantly associated with an unfavorable survival outcome in patients with colonic adenocarcinoma (Hazard ratio $[\mathrm{HR}]=2.15, \mathrm{P}=0.020)$, lung adenocarcinoma $(\mathrm{HR}=3.34, \mathrm{P}=0.030)$, and glioblastoma $(\mathrm{HR}=1.99, \mathrm{P}=0.034)$ and glioma $(\mathrm{HR}=1.56, \mathrm{P}=0.026)$, suggesting that SAMHD1 may contribute to chemoresistance in these cancers (Fig. 8A-8D; Fig. S1D-1G). Using a nude mice model, we further explored the effect of inhibition of the USP7-SAMDH1 axis on chemosensitivity. Consequently, shSAMHD1 cells were more sensitive to cisplatin when compared to shNC cells as determined by tumor volume and weight (Fig. 8E-8G). Importantly, simultaneous administration of P5091 sensitized cancer cells to cisplatin chemotherapy. Taken together, the findings suggest that SAMHD1 may be a potential prognostic biomarker in the adjuvant and neoadjuvant setting, and that the combination of cytotoxic chemotherapy and USP7 inhibition might overcome chemoresistance in various cancers (Fig. 8H).

\section{Discussion}

The observations in this study have demonstrated that SAMHD1 is associated with and stabilized by the deubiquitinase USP7 at K421. Further, SAMHD1 is upregulated in various early-stage human carcinomas and positively correlated with USP7. Furthermore, USP7-stabilized SAMHD1 promotes DDR by interacting with DSB initiator CtIP, thus resulting in DNA damage repair induced by ROS and genotoxic insults. Importantly, USP7 inhibitor sensitizes cancer cells to chemotherapy by decreasing SAMHD1, suggesting USP7-SAMHD1-CtIP axis as a potential target for improvement of chemosensitivity.

Tumorigenesis is accompanied by up-regulation of ROS and accumulation of DNA damage, and the former can induce DNA damage and activate the DNA damage response (DDR) signaling ${ }^{27,28}$.SAMHD1 reportedly promotes DNA end resection to facilitate DNA DSB repair by interacting with CtIP independent of its dNTP hydrolysis ability ${ }^{12}$. In addition, SAMHD1 facilitates degradation of nascent DNA at stalled replication forks in the replication stress response $\mathrm{e}^{29}$. Our findings in human cancerous tissues suggest that SAMHD1 might be implicated in early-stage 
carcinomas to overcome elevated DNA damage and oncogenic stress. In eukaryotic cells, ubiquitination plays an essential role in the assembly as well as disassembly of DDR factors at break sites ${ }^{30,31}$. Both ROS and genotoxic insults, such as cisplatin or doxorubicin, may increase SAMHD1 by inducing its protein deubiquitination and promote the interaction of SAMHD1 with CtIP for DDR. These findings have indicated a novel deubiqutination regulation of SAMHD1 during tumorigenesis.

SAMHD1 has been known as an effector in innate immunity, a restrictor for retroviruses, and a regulator in the cell cycle, which largely depends on its ubiquitination regulatory mechanism ${ }^{32}$. To escape restriction by SAMHD1, HIV-2 and SIV viruses evolved viron-associated Vpx protein degrades SAMHD1 through proteasome pathway via hijacking the Cul4A/DDB1/DCAF1 E3 ligase $^{33}$. It has been recently reported that TRIM21 is the E3 ubiquitin ligase of SAMHD1 for its degradation in enterovirus 71 (EV71) infection ${ }^{34}$. However, the specific deubiquitinase of SAMHD1 still remains unclear. In this study, we have found for the first time that USP7, a pivotal deubiquitinase, directly interacts with the HD domain of SAMHD1 and stabilizes SAMHD1 from degradation through the proteasomal pathway. Colocalization of USP7 and SAMHD1 has been further confirmed by confocal microscopy. Specifically, USP7 increases expression and prolongs the half-life of SAMHD1 protein by reducing its K48-linked polyubiquitination at K421. These findings provide further insight into potential specific regulation of SAMHD1 dysfunction.

A variety of chemotherapeutic agents induce DSB to kill tumor cells, while overexuberant DDR contributes to chemoresistance and worse prognosis in various tumor types ${ }^{35,36}$. The involvement of SAMHD1 in DNA DSB repair suggests a potential role of SAMHD1 in regulating chemosensitivity. Indeed, SAMHD1 knockdown significantly increases chemosensitivity to cisplatin and doxorubicin in tumor cells. Multiple chemotherapeutic agents induce DNA damage and apoptosis in cancer cells $^{37,38}$. Under the treatment of chemotherapy drugs, SAMHD1 significantly inhibits apoptosis independent of its dNTP hydrolase activity. Several previous studies have obtained similar results in this regard. For example, targeting SAMHD1 by the 
Vpx protein has been found to benefit cytarabine therapy for hematological malignancies ${ }^{39}$. Moreover, SAMHD1 expression levels determine acute lymphoblastic leukemia cell response to nelarabine ${ }^{40}$.Therefore, these findings, along with our observations, suggest that SAMHD1 is a potential therapeutic target for improving chemotherapy efficacy in treating malignancies.

Emerging evidence has suggested the implication of USP7 in initiation and development of cancer ${ }^{41,42,43}$. For instance, USP7 deubiquitinates and stabilizes $\mathrm{N}$-myc protein to promote neuroblastoma progression ${ }^{44}$. In addition, USP7 deubiquitinates $\beta$-catenin and activate Wnt signaling to promote colon cancer development ${ }^{45}$. We found that the USP7-SAMHD1 axis contribute to the DDR and chemotherapy insensitivity of cancer cells. Importantly, inhibition of USP7 suppresses chemotherapeutic agents-induced SAMHD1 expression and prevents the formation of SAMHD1 or CtIP foci and their co-localization with $\gamma \mathrm{H} 2 \mathrm{AX}$. Further, activation of the USP7-SAMHD1 axis reduces chemosensitivity and inhibits apoptosis. Attesting to function, USP7 was highly expressed in a variety of tumoral tissues and positively correlated with the SAMHD1 expression. Moreover, the patients with high SAMHD1-expressing tumors who received chemotherapy had unfavorable survival outcomes. Lastly, we have demonstrated that blockade of USP7 by its specific inhibitor P5091 sensitizes cancer cells to cisplatin using a nude mice model, thus further indicating its potential role in chemoresistance.

In summary, we have identified a deubiquitination-dependent regulatory mechanism of the USP7-SAMHD1 axis for sustaining DNA damage response in tumorigenesis and cell survival under genotoxic insults. These findings suggest that SAMHD1 may be a potential biomarker for chemosensitivity, and that combination of chemotherapy and USP7 inhibition may potentially help overcome chemoresistance in various cancer types.

\section{Methods}

\section{Cell culture}

HCT116, SW480, H1299, HEK293, Hela cells were purchased from Cell Bank in Chinese Academy of Sciences Shanghai. HCT116, SW480 and H1299 were 
cultured in RPMI 1640 medium; HEK293 and Hela were cultured in high-glucose Dulbecco's modified Eagle's medium (DMEM), supplemented with10\% fetal bovine serum (FBS)(CLARK, Australia), penicillin (100U), and streptomycin (100g/ml).

\section{Antibodies and reagents}

Antibodies used in this study includeUSP7(Western blot: \#4833, CST; ab4080, Abcam; Immunohistochemistry: NB100-513, NOVUS), SAMHD1 (Western blot:\#12361, $\quad$ CST; $\quad$ IP: ab245389, Immunohistochemistry/Immunofluorescence: ab128107, Abcam), CtIP (Western blot: \#9201, CST; Immunofluorescence:NB100-79810), $\beta$-actin (AC004, ABclonal), Flag (SG4110-16, Shanghai Genomics Technology), Myc (SG4110-18, Shanghai Genomics Technology), HA (\#3724, CST), Ubiquitin (\#3933S, CST), PARP (\#9532, CST),Cleaved PARP (\#5625, CST), Caspase3 (\#9662, CST), Cleaved Caspase3 (\#9664, CST), Phospho-Histone H2A.X (Ser139) (\#9718, CST; \#80312, CST).

CHX (S7418), P5091 (S7132), Cisplatin (S1166), Doxorubicin (Adriamycin) $\mathrm{HCl}$ (S1208) and MG132 (S2619) were purchased from Selleck. PI (propidium iodide, ST511) and NAC (S0077) were from Beyotime. IPTG (I6758) were from Sigma-Aldrich.

\section{Plasmid constructions, transfection and lentivirus infection}

SAMHD1 and USP7 expression plasmids were purchased from Shanghai GeneChem Company. Mutagenesis (SAMHD1 K421R, SAMHD1 K492R, SAMHD1 K494R, SAMHD1 H206A/D207A and USP7C223S) was performed based on Quick Change Site-Directed Mutagenesis Kit (Stratagene, La Jolla, CA). Specific siRNAs for USP7 were purchased from RiboBio Co., Ltd., Guangzhou, China. The plasmids were confirmed by sequencing and transfected into HEK293, HCT116 or SW480 cells using Lipofectamine 3000 reagent (Thermo Fisher Scientific, USA) according to the manufacturer's instructions. Cells were harvested 48 hours after transfection. For lentiviral production and infection, control shRNA (shNC) lentivirus, shRNA against SAMHD1 (shSAMHD1) lentivirus, shRNA against USP7(shUSP7) lentivirus, Flag-vector overexpression lentivirus (Flag) and SAMHD1 overexpression lentivirus 
(Flag-SAMHD1) were purchased from Shanghai GeneChem Company. Stably infected cell lines were selected by puromycin after lentivirus infection for 5 days.

\section{Mass spectrometry}

We overexpressed Flag-SAMHD1 in HEK-293 cells and collected the cells after 48 hours. IP lysis was used for cell lysis, and Flag-beads was added to the protein lysis solution. The solution was mixed overnight in a chromatography cabinet at $4^{\circ} \mathrm{C}$, which was centrifuged and washed with PBS at $4{ }^{\circ} \mathrm{C}$ for 3 times. The beads were boiled with $2 \times$ loading in a water bath for 10 minutes. The immunoprecipitated protein were subjected to western blot and excised from the gel for digestion and mass spectrometry. Interaction protein and peptide identifications were conducted by database search.

\section{Western blot analysis and Co-immunoprecipitation (Co-IP)}

Cells were lysed for 30 min on ice with IP lysis buffer supplemented with protease inhibitor cocktails, then the lysed protein was harvested by centrifugation at $15,000 \mathrm{rpm}$ for $20 \mathrm{~min}$ at $4^{\circ} \mathrm{C}$. Protein concentration was assessed by $\mathrm{G} 250$ and $40 \mu \mathrm{g}$ of cell lysate were adopted for samples. Protein samples were separated on $8 \%, 10 \%$ or $12 \%$ SDS PAGE and transferred to PVDF membrane (Millipore, IPVH00010) for two hours at 80V.After block in 5\% BSA in TBST for one hour at room temperature, the membranes were probed with specific primary antibodies at $4^{\circ} \mathrm{C}$ overnight. The membranes were then washed with TBST three times followed by incubation with HRP-conjugated secondary antibody at room temperature for two hours. After three washes, bands were detected by enhanced chemi-luminescence detection kit (Thermo Fisher Scientific, 32106) and visualized via the DNR western blot detection system.

Cells were lysed with IP lysis buffer $(25 \mathrm{mM}$ Tris, $\mathrm{pH} 7.6,150 \mathrm{mM} \mathrm{NaCl}$, $1 \%$ Nonidet P-40, $1 \mathrm{mM}$ EDTA), and $1 \mathrm{mg}$ protein was incubated with antibody and protein A/G-Sepharose (Santa Cruz, sc-2003) on a rocking platform overnight at $4^{\circ} \mathrm{C}$. Next, the beads were harvested by centrifugation for $5 \mathrm{~min}$ at $700 \mathrm{~g}$ at $4{ }^{\circ} \mathrm{C}$ and the supernatant was removed. The beads were then resuspended in IP lysis buffer and repeatedly inverted on a rocking platform for $10 \mathrm{~min}$. This wash was repeated three times to remove the nonspecific binding protein and obtain purified protein 
complexes that are bound to the antibody-coated beads. Finally, the beads were resuspended with loading buffer for Western blot analysis.

\section{GST-pulldown}

The bacterial expression constructs (pGEX-4T-1) containing the target genes (SAMHD1 or USP7) were transformed into BL21-competent cells (Takara). Cells were induced to overexpress the GST-fusion protein by $1 \mathrm{mM}$ IPTG for $3 \mathrm{~h}$ while shaking at $30{ }^{\circ} \mathrm{C}$. Cells were resuspended in bacterial lysis (PBS containing $1 \mathrm{mM}$ PMSF, $5 \mathrm{mM} \beta$-mercaptoethanol,0.5\% TritonX-100 and $2 \mathrm{mM}$ EDTA), followed by ultrasonication. The proteins were purified by a single step using glutathione bead according to the manufacturer's protocol (Promega Science). In vitro transcription and translation of SAMHD1 or USP7 proteins were performed by T7-TNT Kits (Promega, L1170) in accordance with the manufacturer's instructions. GST pulldown assays were performed as previously described ${ }^{46}$.

\section{Immunofluorescent analysis}

For immunofluorescence in cultured cells, the culture medium was discarded and washed 4 times with PBS, then subjected to treatment with tissue fixative for 20 minutes. The cells were then washed 3 times with PBS and treated by $0.25 \%$ TritonX-100 for 15 minutes. The samples were washed 3 times with PBS before block with 5\% BSA for 1 hour, and then incubated the primary antibody overnight at $4^{\circ} \mathrm{C}$. After three times wash with PBS the next day, the corresponding fluorescent secondary antibody (1:400) was incubated at room temperature for $1 \mathrm{~h}$ in the dark. After three times wash with PBS, the samples were stained with DAPI for 5 minutes and mount upside down on the glass slide, which was protected from light and dry for observation under the laser confocal microscope in a dark room.

For tissue immunofluorescence, the colonic tissues were fixed in $4 \%$ paraformaldehyde overnight and then sections $(100 \mu \mathrm{m})$ were incubated in 5\% normal donkey serum in PBS containing 0.5\% Triton X-100 to block nonspecific binding for $1 \mathrm{~h}$ at room temperature. Primary antibody against USP7 and SAMHD1 were diluted in PBS containing $0.1 \%$ Triton $\mathrm{X}-100$ and $1 \%$ donkey serum and incubated with sections overnight at $4{ }^{\circ} \mathrm{C}$. The tissue sections were subjected to anti-Rabbit-Cy2 or 
anti-mouse-Cy2 secondary antibodies (1:250, Jackson), followed by DAPI staining. Then tissue sections were then mounted and observed using a confocal laser scanning microscopy (Zeiss LSM880).

\section{RNA isolation and quantitative real-time PCR}

Total RNA was isolated from cells using Trizol reagent. Reverse transcription was performed using a PrimeScript RT Reagent Kit (Takara, RR037A), according to the manufacturer's instructions. The cDNA was quantified by quantitative real-time PCR with SYBR®Premix Ex Taq ${ }^{\mathrm{TM}}$ II (Takara, RR820A) on Mx3000P instrument (Agilent StrataGene). The sequences of SAMHD1 primers were Forward: TGCAGAGCAGCTGATTCGAG; Reverse: ATAACATCGCCATCCTGCGG. SAMHD1 mRNA expression was calculated relative to expression of the housekeeping gene $\beta$-actin using Stratagene Mx3000P software.

\section{Cell proliferation assay}

HCT116 or SW480 cells were seeded in triplicate at a density of $1 \times 10^{4}$ cells per well into 96-well plates. After 24-hour incubation in complete RPMI 1640 with 10\% FBS, cells were exposed to Cisplatin or Doxorubicin at different concentrations. Upon measurement, RPMI 1640 medium and CCK8(Cell Counting Kit-8) staining solution was added to cells at each well for 2 hours at $37^{\circ} \mathrm{C}$. The absorbance was measured at450 nm daily using an absorbance reader (TECAN, Switzerland). The percentage of cell survival was then calculated.

\section{Flow cytometric analysis}

To investigate cell apoptosis, HCT116 or SW480 cells were treated with Cisplatin for $24 \mathrm{~h}$, followed by incubation with PI and FITC-Annexin V (BD Phamingen, 556547). In addition, HCT116 cells were treated with Doxorubicin for 24 $\mathrm{h}$, followed by incubation with APC and 7-AAD (KeyGEN BioTECH, KGA1026). The percentage of apoptotic cells was then measured according to the manufacturer's protocol.

\section{Immunohistochemistry}

The tissue microarrays of early stage of colon adenocarcinoma (HCol-Ade060CS1-01), lung adenocarcinoma (HLugA150CS03), thyroid carcinoma 
(HThyP120CS02) were purchased from Shanghai Outdo Biotech Company, China. After deparaffinizing in xylene and rehydrating in graded ethanol, tissue microarrays were immersed in citrate buffer for antigen retrieval. Endogenous peroxidase was quenched using $3 \%$ hydrogen peroxide for $30 \mathrm{~min}$. To decrease the nonspecific staining, $10 \%$ normal goat serum was subsequently used to block tissue collagen for $30 \mathrm{~min}$. Tissue sections were then incubated with antibody anti-SAMHD1 (ab128107, 1:2000, Abcam) or USP7 (NB100-513, 1:1000, NOVUS) for 60 minutes at room temperature $\left(24-27^{\circ} \mathrm{C}\right)$. After that, biotinylated secondary antibody and streptavidin-biotin peroxidase were used to incubate tissue sections for $10 \mathrm{~min}$ each in turn. Slides were stained with DAB chromogenic reagent for $60 \mathrm{~s}$, afterwards counterstained with hematoxylin. UltraSensitive ${ }^{\mathrm{TM}}$ SPIHC Kit(KIT-9720,Maixin Inc., Fujian, China) were used in this experiment.

The stained sections were reviewed and scored by two investigators independently and the different scoring was resolved after discussion. We adopted a semi-quantitative scoring method to assess the expression of SAMHD1 and USP7. The staining intensity was divided into 0 (no staining), 1 (weak staining), 2 (moderate) and 3 (strong). The percentage of cells stained was categorized as $0(0-5 \%), 1$ (6-25\%), 2 (26-50\%), 3 (51-75), and 4 (76\%-100\%). The final scores were generated by multiplying the staining intensity by percentage of cells, which were classified as: 0-4, low; 5-8, medium; 9-12, high; respectively.

\section{Tumor xenografts}

We use the nude mouse model to further analyze the role of USP7-SAMHD1 axis in colon cancer chemosensitivity. A total of 48 female nude mice (BALB/cA-nu Mice) at 4 weeks of age were subcutaneously inoculated $5 \times 10^{6}$ HCT116 shNC and HCT116 shSAMHD1 cells. After 1 week, the mice were randomly divided into shNC, shNC+cisplatin, shSAMHD1, shSAMHD1+cisplatin, P5091, and P5091+cisplatin groups, respectively. Cisplatin and P5091 were administered twice a week at a concentration of $2 \mathrm{mg} / \mathrm{kg}$ and $5 \mathrm{mg} / \mathrm{kg}$, respectively). The tumor size was measured daily. The tumor volume was calculated (length $\times$ width $^{2}$ ). The mice were sacrificed after 25 days, and the tumors were removed for subsequent analyses. 


\section{Statistical analysis}

SPSS version 20.0 (SPSS Inc., Chicago IL, USA) was used for statistical analyses. Two-tailed Student's t test was used for continuous variables. Spearman's rank correlation test was used to evaluate the correlation between SAMHD1 and USP7 expression. Survival outcomes were assessed by the Kaplan-Meier method, and the log-rank test was used to compare the differences between the groups. The Cox proportional hazards model was used to investigate the association of survival time and SAMHD1 expression. A P $<0.05$ was considered statistically significant.

Acknowledgement: We thank Professor Shi Wei (University of Alabama at Birmingham, Birmingham, USA.) for revising the manuscript. Funding: This work was supported by the key project of the National Natural Science Foundation (82030091), National Key R\&D Program of China (2016YFC1302400), the Ministry of education innovation team development plan (IRT_17R107), the key project of LiaoNing Science Foundation (2019JH2/10300, Z18-4-021), the National Natural Science Foundation (82073089), the Natural Science Foundation of Liaoning Province (2019-ZD-0748).

Author contributions: L.C., C.X., T.Z. and X.S. conceived and designed the study. J.L., X.D., Q.G., L.Z., N.Z. and L.R. conducted the cell experiments. X.W., F.Y. and Y.Z. performed the animal experiments. D.L., C.L. and C.D. conducted the qPCR experiments. Z.W., Z.L., X.W., H.X. and Y.G. carried out the immunohistochemistry assays. H.L., X.L., Y.Y., W.G. and Z.W. performed the statistical analysis. B.J., X.W., N.B. and Y.F. conducted the remaining in vitro experiments. J.L., M.M., Q.K. and J.W. wrote the manuscript.

Competing interests: The authors declare no competing interests. 


\section{Reference}

1. Jackson SP, Bartek J. The DNA-damage response in human biology and disease. Nature 2009, 461(7267): 1071-1078.

2. Roos WP, Thomas AD, Kaina B. DNA damage and the balance between survival and death in cancer biology. Nat Rev Cancer 2016, 16(1): 20-33.

3. O'Connor MJ. Targeting the DNA Damage Response in Cancer. Mo/ Cel/ 2015, 60(4): 547-560.

4. Lord CJ, Ashworth A. The DNA damage response and cancer therapy. Nature 2012, 481(7381): 287-294.

5. Goldstein M, Kastan MB. The DNA damage response: implications for tumor responses to radiation and chemotherapy. Annu Rev Med 2015, 66: 129-143.

6. Chen S, Bonifati S, Qin Z, St Gelais C, Wu L. SAMHD1 Suppression of Antiviral Immune Responses. Trends Microbio/ 2019, 27(3): 254-267.

7. Chen Z, Zhang L, Ying S. SAMHD1: a novel antiviral factor in intrinsic immunity. Future Microbio/ 2012, 7(9): 1117-1126.

8. Coggins SA, Mahboubi B, Schinazi RF, Kim B. SAMHD1 Functions and Human Diseases. Viruses 2020, 12(4).

9. Majer C, Schussler JM, Konig R. Intertwined: SAMHD1 cellular functions, restriction, and viral evasion strategies. Med Microbiol Immuno/ 2019, 208(3-4): 513-529.

10. Mauney $\mathrm{CH}$, Hollis T. SAMHD1: Recurring roles in cell cycle, viral restriction, cancer, and innate immunity. Autoimmunity 2018, 51(3): 96-110.

11. Zhang Z, Zheng L, Yu Y, Wu J, Yang F, Xu Y, et al. Involvement of SAMHD1 in dNTP homeostasis and the maintenance of genomic integrity and oncotherapy (Review). Int J Onco/ 2020, 56(4): 879-888.

12. Daddacha W, Koyen AE, Bastien AJ, Head PE, Dhere VR, Nabeta GN, et al. SAMHD1 Promotes DNA End Resection to Facilitate DNA Repair by Homologous Recombination. Cell Rep 2017, 20(8): 1921-1935.

13. Scully R, Panday A, Elango R, Willis NA. DNA double-strand break repair-pathway choice in somatic mammalian cells. Nat Rev Mol Cell Bio/2019, 20(11): 698-714.

14. Mattiroli F, Vissers JH, van Dijk WJ, Ikpa P, Citterio E, Vermeulen W, et al. RNF168 ubiquitinates $\mathrm{K} 13-15$ on H2A/H2AX to drive DNA damage signaling. Ce// 2012, 150(6): 
1182-1195.

15. Thorslund T, Ripplinger A, Hoffmann S, Wild T, Uckelmann M, Villumsen B, et al. Histone $\mathrm{H} 1$ couples initiation and amplification of ubiquitin signalling after DNA damage. Nature 2015, 527(7578): 389-393.

16. Antao AM, Tyagi A, Kim KS, Ramakrishna S. Advances in Deubiquitinating Enzyme Inhibition and Applications in Cancer Therapeutics. Cancers (Basel) 2020, 12(6)

17. Bojagora A, Saridakis V. USP7 manipulation by viral proteins. Virus Res 2020, 286: 198076

18. Pozhidaeva A, Bezsonova I. USP7: Structure, substrate specificity, and inhibition. DNA Repair (Amst) 2019, 76: 30-39.

19. Rawat R, Starczynowski DT, Ntziachristos P. Nuclear deubiquitination in the spotlight: the multifaceted nature of USP7 biology in disease. Curr Opin Cell Bio/ 2019, 58: 85-94.

20. Valles GJ, Bezsonova I, Woodgate R, Ashton NW. USP7 Is a Master Regulator of Genome Stability. Front Cell Dev Bio/2020, 8: 717.

21. Ballana E, Este JA. SAMHD1: at the crossroads of cell proliferation, immune responses, and virus restriction. Trends Microbio/ 2015, 23(11): 680-692.

22. Moloney JN, Cotter TG. ROS signalling in the biology of cancer. Semin Cel/ Dev Bio/2018, 80: 50-64.

23. Srinivas US, Tan BWQ, Vellayappan BA, Jeyasekharan AD. ROS and the DNA damage response in cancer. Redox Bio/2019, 25: 101084.

24. Harrigan JA, Jacq X, Martin NM, Jackson SP. Deubiquitylating enzymes and drug discovery: emerging opportunities. Nat Rev Drug Discov 2018, 17(1): 57-78.

25. Cui Q, Wang JQ, Assaraf YG, Ren L, Gupta P, Wei L, et al. Modulating ROS to overcome multidrug resistance in cancer. Drug Resist Updat 2018, 41: 1-25.

26. Marullo R, Werner E, Degtyareva N, Moore B, Altavilla G, Ramalingam SS, et al. Cisplatin induces a mitochondrial-ROS response that contributes to cytotoxicity depending on mitochondrial redox status and bioenergetic functions. PLoS One 2013, 8(11): e81162.

27. de Sa Junior PL, Camara DAD, Porcacchia AS, Fonseca PMM, Jorge SD, Araldi RP, et al. The Roles of ROS in Cancer Heterogeneity and Therapy. Oxid Med Cell Longev 2017, 2017: 2467940 
28. Gorrini C, Harris IS, Mak TW. Modulation of oxidative stress as an anticancer strategy. Nat Rev Drug Discov 2013, 12(12): 931-947.

29. Coquel F, Silva MJ, Techer H, Zadorozhny K, Sharma S, Nieminuszczy J, et al. SAMHD1 acts at stalled replication forks to prevent interferon induction. Nature 2018, 557(7703): $57-61$

30. Dybas JM, Herrmann C, Weitzman MD. Ubiquitination at the interface of tumor viruses and DNA damage responses. Curr Opin Viro/ 2018, 32: 40-47.

31. Le J, Perez E, Nemzow L, Gong F. Role of deubiquitinases in DNA damage response. DNA Repair (Amst) 2019, 76: 89-98.

32. Li M, Zhang D, Zhu M, Shen Y, Wei W, Ying S, et al. Roles of SAMHD1 in antiviral defense, autoimmunity and cancer. Rev Med Virol 2017, 27(4).

33. Romani B, Cohen EA. Lentivirus Vpr and Vpx accessory proteins usurp the cullin4-DDB1 (DCAF1) E3 ubiquitin ligase. Curr Opin Viro/ 2012, 2(6): 755-763.

34. Li Z, Huan C, Wang H, Liu Y, Liu X, Su X, et al. TRIM21-mediated proteasomal degradation of SAMHD1 regulates its antiviral activity. EMBO Rep 2020, 21(1): e47528.

35. Desai A, Yan Y, Gerson SL. Advances in therapeutic targeting of the DNA damage response in cancer. DNA Repair (Amst) 2018, 66-67: 24-29.

36. Trenner A, Sartori AA. Harnessing DNA Double-Strand Break Repair for Cancer Treatment. Front Oncol 2019, 9: 1388

37. Carneiro BA, El-Deiry WS. Targeting apoptosis in cancer therapy. Nat Rev Clin Oncol 2020, 17(7): 395-417.

38. Hosoya N, Miyagawa K. Targeting DNA damage response in cancer therapy. Cancer SCi 2014, 105(4): 370-388.

39. Herold N, Rudd SG, Ljungblad L, Sanjiv K, Myrberg IH, Paulin CB, et al. Targeting SAMHD1 with the $V p x$ protein to improve cytarabine therapy for hematological malignancies. Nat Med 2017, 23(2): 256-263.

40. Rothenburger T, McLaughlin KM, Herold T, Schneider C, Oellerich T, Rothweiler F, et al. SAMHD1 is a key regulator of the lineage-specific response of acute lymphoblastic leukaemias to nelarabine. Commun Bio/2020, 3(1): 324

41. He Y, Wang S, Tong J, Jiang S, Yang Y, Zhang Z, et al. The deubiquitinase USP7 stabilizes Maf proteins to promote myeloma cell survival. J Biol Chem 2020, 295(7): 2084-2096. 
42. Qi SM, Cheng G, Cheng XD, Xu Z, Xu B, Zhang WD, et al. Targeting USP7-Mediated Deubiquitination of MDM2/MDMX-p53 Pathway for Cancer Therapy: Are We There Yet? Front Cell Dev Biol 2020, 8: 233.

43. Sun X, Ding Y, Zhan M, Li Y, Gao D, Wang G, et al. Usp7 regulates Hippo pathway through deubiquitinating the transcriptional coactivator Yorkie. Nat Commun 2019, 10(1): 411.

44. Tavana O, Li D, Dai C, Lopez G, Banerjee D, Kon N, et al. HAUSP deubiquitinates and stabilizes N-Myc in neuroblastoma. Nat Med 2016, 22(10): 1180-1186.

45. Novellasdemunt L, Foglizzo V, Cuadrado L, Antas P, Kucharska A, Encheva V, et al. USP7 Is a Tumor-Specific WNT Activator for APC-Mutated Colorectal Cancer by Mediating beta-Catenin Deubiquitination. Cell Rep 2017, 21(3): 612-627.

46. Guo QQ, Wang SS, Zhang SS, Xu HD, Li XM, Guan Y, et al. ATM-CHK2-Beclin 1 axis promotes autophagy to maintain ROS homeostasis under oxidative stress. EMBO J2020, 39(10): e103111.

Figure legends

Figure 1. ROS induces deubiquitination and increases of SAMHD1 for DNA damage repair.

A-F, Representative sections and semiquantitative analyses of SAMHD1 expression in early-stage (stage I and II) colonic adenocarcinoma (A and B, n=30), lung adenocarcinoma ( $C$ and $\mathrm{D}, \mathrm{n}=48$ ), and thyroid carcinoma ( $\mathrm{E}$ and $\mathrm{F}, \mathrm{n}=58$ ). Data are expressed as mean \pm SEM. $* * * \mathrm{P}<0.001$ (Mann-Whitney test). G, Western blot analysis of SAMHD1 in $\mathrm{H} 1299$ cells without and with $\mathrm{H}_{2} \mathrm{O}_{2}(25 \mu \mathrm{M})$ treatment. $\mathbf{H}$, Western blot analysis of SAMHD1 in H1299 cells in the presence and absence of NAC (3 mM) pretreatment, with and without $\mathrm{H}_{2} \mathrm{O}_{2}(25 \mu \mathrm{M})$ stimulation for $1 \mathrm{~h}$. I, The lysates of HCT116 cells without and with $1 \mathrm{~h} \mathrm{H}_{2} \mathrm{O}_{2}(25 \mu \mathrm{M})$ stimulation were 
immunoprecipitated with control IgG or anti-SAMHD1 antibody followed by immunoblotting with ubiquitin antibody. J, The lysates of HCT116 cells with and without $1 \mathrm{~h} \mathrm{H}_{2} \mathrm{O}_{2}(25 \mu \mathrm{M})$ stimulation were immunoprecipitated with the IgG control or anti-SAMHD1 antibody followed by immunoblotting with SAMHD1 and CtIP antibodies. $\mathbf{K}$ and $\mathbf{L}$, HCT116 cells with and without $1 \mathrm{~h} \mathrm{H}_{2} \mathrm{O}_{2}(500 \mu \mathrm{M})$ stimulation were stained with the anti-SAMHD1 or anti-CtIP and anti- $\gamma \mathrm{H} 2 \mathrm{AX}$ antibodies, DAPI, respectively, for confocal immunofluorescence analysis. Scale bar, $10 \mu \mathrm{m}$.

\section{Figure 2. USP7 interacts with SAMHD1 at the HD domain}

A, Flag-tagged SAMHD1 was transfected into the HEK293 cells and immunoprecipitated with an anti-Flag antibody. SAMHD1 was purified with a Flag peptide followed by mass spectrometry analysis. B, The lysates of the HCT116 cells were immunoprecipitated with the IgG control or anti-SAMHD1 antibody followed by immunoblotting with USP7 and SAMHD1 antibodies. C, A Flag-tagged USP7 plasmid was transfected into the HCT116 cells as indicated. The total lysates were subjected to IP and Western blot with the anti-Flag antibody. D and E, SAMHD1 interacts with USP7 in vitro demonstrated by a GST pull-down assay. Recombinant human SAMHD1 or USP7 was incubated with bacterially expressed GST-USP7or GST-SAMHD1. F, Schematic plot for constructing truncated plasmids of SAMHD1 lacking different domains including NtD (1-34), SAM (35-114), HD (115-583) or CtD (584-626), respectively. G, USP7 interacts with the HD domain of SAMHD1. Truncated plasmid of SAMHD1 lacking different domains including NtD, SAM, HD or CtD were constructed for GST pull-down assay with recombinant USP7, respectively. H, SAMHD1 colocalized with USP7 in Hela cells by immunofluorescence assay. Scale bar, $10 \mu \mathrm{m}$.

Figure 3. USP7 stabilizes the expression of SAMHD1in HCT116 cells.

A, Western blot analysis demonstrating gradually increased SAMHD1 protein levels with increased amounts of Flag-USP7 plasmids transfected. B, The effect of three target sequences of siRNA-USP7 on the expression of SAMHD1 48h after 
transfection. C, Western blot analysis of SAMHD1 with or without P5091 treatment for $24 \mathrm{~h}$ at different concentrations. D and E, Levels of SAMHD1 expression after different durations of CHX $(20 \mu \mathrm{M})$ administration with or without MG132 $(20 \mu \mathrm{M})$ treatment. Data are shown as mean \pm SEM $(* * \mathrm{P}<0.01)$. F and G, SAMHD1 expression after different durations of CHX $(20 \mu \mathrm{M})$ administration in the cells $48 \mathrm{~h}$ after transfected with HA vector, HA-USP7 WT or HA-USP7 C223S mutant plasmids. Data are shown as mean \pm SEM $(* * * \mathrm{P}<0.001)$. H and I, SAMHD1 expression after different durations of CHX $(20 \mu \mathrm{M})$ administration, with or without USP7 knockdown. Data are shown as mean \pm SEM $(* * \mathrm{P}<0.01)$. J and $\mathbf{K}$, SAMHD1 expression after different durations of CHX $(20 \mu \mathrm{M})$ administration, with or without P5091 $(20 \mu \mathrm{M})$ pretreatment. Data are shown as mean $\pm \operatorname{SEM}(* * * \mathrm{P}<0.001)$.

\section{Figure 4. USP7 deubiquitinates SAMHD1 K48-linked polyubiquitination at} K421.

A, The HCT116 cells were lysed $48 \mathrm{~h}$ after transfection with or without Flag-USP7, and then immunoprecipitated with the anti-SAMHD1 antibody or IgG control, followed by immunoblotting with the ubiquitin antibody. B, The lysates of HCT116 cells with or without USP7 knockdown were immunoprecipitated with the anti-SAMHD1 antibody or IgG control, followed by immunoblotting with the

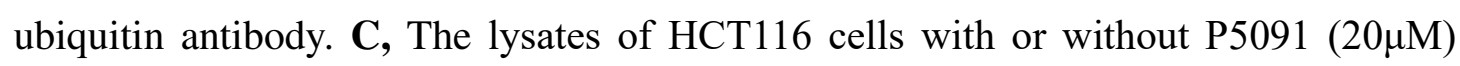
pretreatment for $24 \mathrm{~h}$ were immunoprecipitated with the anti-SAMHD1 antibody or IgG control, followed by immunoblotting with the ubiquitin antibody. D, The HCT116 cells were lysed $48 \mathrm{~h}$ after transfection with Flag-SAMHD1, HA-Ub and Myc/Myc-USP7 WT/Myc-USP7 C223S, respectively, and then immunoprecipitated with the Flag antibody, followed by immunoblotting with the HA antibody. E-G, The lysates of HCT116 cells were lysed $48 \mathrm{~h}$ after transfection with Flag-SAMHD1 WT/Flag-SAMHD1 K421R/Flag-SAMHD1 K492R/Flag-SAMHD1 K494R, HA-Ub and Myc/Myc-USP7, and then immunoprecipitated with the Flag antibody, followed by immunoblotting with the HA antibody. H, The HCT116 cells were lysed $48 \mathrm{~h}$ after transfection with Flag-SAMHD1, Myc-USP7 and HA-Ub WT/K0/K48/K63, 
respectively, and then immunoprecipitated with the Flag antibody, followed by immunoblotting with the HA antibody.

Figure 5. SAMHD1 increases cell survival and reduces apoptosis under genotoxic insults

A-D, HCT116 shNC and shSAMHD1 cells or shSAMHD1-Flag and shSAMHD1-Flag-SAMHD1 cells were treated with cisplatin or doxorubicin at different concentrations for $24 \mathrm{~h}$. Cell viability was assessed by CCK8 assay. E and F, HCT116 shNC and shSAMHD1 cells or shSAMHD1-Flag and shSAMHD1-Flag-SAMHD1 cells were treated with cisplatin $(20 \mu \mathrm{M})$ for $24 \mathrm{~h}$ followed by staining with PI and FITC-Annexin V, and analyzed by fluorescence-activated cell sorting (FACS). Bar graph represents percentage of apoptotic cells from three independent experiments. G, HCT116 and SW480 shSAMHD1-Flag, shSAMHD1-Flag-SAMHD1 WT and shSAMHD1-Flag-SAMHD1 H206A/D207A cells were treated with cisplatin $(20 \mu \mathrm{M})$ for $24 \mathrm{~h}$ followed by staining with PI and FITC-Annexin V, and analyzed by FACS. Bar graph represents percentage of apoptotic cells from three independent experiments. H, HCT116 cells with or without cisplatin $(5 \mu \mathrm{M})$ stimulation for $4 \mathrm{~h}$ were stained with anti-SAMHD1 and anti- $\gamma \mathrm{H} 2 \mathrm{AX}$ antibodies, DAPI, respectively, for immunofluorescence analysis. Scale bar, 10 $\mu \mathrm{m}$. I and J, HCT116 shNC and shSAMHD1 cells or shSAMHD1-Flag and shSAMHD1-Flag-SAMHD1 cells were treated with cisplatin $(5 \mu \mathrm{M})$ for $12 \mathrm{~h}$ followed by western blot analysis of PARP, Cleaved-PARP, Caspase 3, Cleaved-Caspase 3, and $\gamma \mathrm{H} 2 \mathrm{AX}$. Data are expressed as means \pm SEM. NS, no significance; $* \mathrm{P}<0.05 ; * * \mathrm{P}<0.01 ; * * * \mathrm{P}<0.001$.

Figure 6. The USP7-SAMHD1 axis modulates cell survival and apoptosis under genotoxic insults

A, The lysates of HCT116 cells with or without cisplatin $(5 \mu \mathrm{M})$ stimulation for $0.5 \mathrm{~h}$ or $1 \mathrm{~h}$ were immunoprecipitated with the anti-SAMHD1 antibody followed by immunoblotting with the SAMHD1 and USP7 antibodies. B, Western blot analysis of 
SAMHD1 in HCT116 cells pretreated with or without P5091 $(20 \mu \mathrm{M})$ and stimulated with and without cisplatin $(5 \mu \mathrm{M})$ for $1 \mathrm{~h}$. C and D, HCT116 cells with or without P5091 $(20 \mu \mathrm{M})$ pretreatment were stimulated with or without cisplatin $(5 \mu \mathrm{M})$ for $4 \mathrm{~h}$, and then stained with the anti-SAMHD1 or anti-CtIP and anti- $\gamma \mathrm{H} 2 \mathrm{AX}$ antibodies, DAPI, respectively, for immunofluorescence analysis. Scale bar, $10 \mu \mathrm{m}$. E and F, HCT116 shNC and shUSP7 cells or shUSP7-Flag and shUSP7-Flag-SAMHD1 cells were treated with cisplatin at different concentrations for $24 \mathrm{~h}$. Cell viability was assessed by CCK8 assay. G and H, HCT116 shNC and shUSP7 cells or shUSP7-Flag and shUSP7-Flag-SAMHD1 cells were treated with cisplatin $(20 \mu \mathrm{M})$ for $24 \mathrm{~h}$ followed by staining with PI and FITC-Annexin V, and analyzed by FACS. Bar graph represents percentage of apoptotic cells from three independent experiments. I, HCT116 cells with or without P5091 $(20 \mu \mathrm{M})$ administration were treated with cisplatin $(20 \mu \mathrm{M})$ for $24 \mathrm{~h}$ followed by staining with PI and FITC-Annexin V, and analyzed by FACS. Bar graph represents percentage of apoptotic cells from three independent experiments. J and K, HCT116 shNC and shUSP7 cells or shUSP7-Flag and shUSP7-Flag-SAMHD1 cells were treated with cisplatin $(5 \mu \mathrm{M})$ for $12 \mathrm{~h}$ followed by western blot analysis of PARP, Cleaved-PARP, Caspase 3, Cleaved-Caspase 3, $\gamma \mathrm{H} 2 \mathrm{AX}$. Data are expressed as mean $\pm \mathrm{SEM} . * \mathrm{P}<0.05 ; * * * \mathrm{P}<0.001$.

\section{Figure 7. USP7 and SAMHD1 are positively correlated in various cancer types.}

A-F, Representative sections and semiquantitative analyses of USP7 expression in colonic adenocarcinoma ( $\mathrm{A}$ and $\mathrm{B}, \mathrm{n}=30$ ), lung adenocarcinoma $(\mathrm{C}$ and $\mathrm{D}, \mathrm{n}=48)$, and thyroid carcinoma (E and $\mathrm{F}, \mathrm{n}=58)$. Data are expressed as mean \pm SEM. $* * * \mathrm{P}<0.001$ (Mann-Whitney test). G-I, Expressions of USP7 and SAMHD1 are positively correlated in consecutive sections of colonic carcinoma $(\mathrm{G})$, lung adenocarcinoma $(\mathrm{H})$ and thyroid carcinoma tissues (I). Results of spearman correlation analyses of $\mathrm{P}$ value and correlation coefficient are shown. $\mathbf{J}$ and $\mathbf{K}$, Correlation of USP7 and SAMHD1 expressions in human lung and thyroid carcinomas in the GEPIA database. L, Colonic adenocarcinoma and peritumoral tissues stained with the anti-SAMHD1 antibody, anti-USP7 antibody and DAPI, respectively, for immunofluorescent analyses. Scale 
bar, $10 \mu \mathrm{m}$.

Figure 8. Inhibition of the USP7-SAMHD1 axis improves chemosensitivity.

A-D, A higher SAMHD1 expression is significantly associated with an unfavorable survival outcome in patients with carcinoma of various organs who received chemotherapy according to The Cancer Genome Atlas (TCGA) data. E-G, Tumor growth assay in nude mice subcutaneously inoculated with shNC or shSAMHD1 cells. The mice were treated with cisplatin, with or without P5091. The images of tumors were acquired (E), and their volume (F) and weight $(\mathrm{G})$ were determined. Data are shown as mean $\pm \mathrm{SD}(\mathrm{n}=5$ for each group). $* \mathrm{P}<0.05 ; * * \mathrm{P}<0.01$. H, A schematic model showing the proposed role of the USP7-SAMHD1 axis in controlling tumor cell survival and chemosensitivity.

\section{Supplementary Figure Legends}

Figure S1. SAMHD1 is highly expressed in carcinomas of various organs and is associated with a worse survival in patients receiving chemotherapy.

A-C, Semiquantitative analyses of SAMHD1 expression in human colonic adenocarcinoma $(\mathrm{A}, \mathrm{n}=30)$, lung adenocarcinoma $(\mathrm{B}, \mathrm{n}=48)$ and thyroid carcinoma tissues $(\mathrm{C}, \mathrm{n}=58)$, and their paired peritumoral tissues. $* * * \mathrm{P}<0.001$ (Mann-Whitney test). D-G, Data from TCGA on the survival outcomes in patients with colonic adenocarcinoma $(n=157)$, lung adenocarcinoma $(n=173)$, glioblasma $(n=123)$ and glioma $(n=292)$ who received chemotherapy.

Figure S2. ROS and genotoxic insults induce deubiquitination and increase SAMHD1 for DNA damage repair.

A, Western blot analysis of SAMHD1 in HCT116 cells with and without $\mathrm{H}_{2} \mathrm{O}_{2}(25 \mu \mathrm{M})$ treatment. B, Western blot analysis of SAMHD1 in HCT116 cells pretreated with or without NAC ( $3 \mathrm{mM})$, followed by $2 \mathrm{~h}$ of $\mathrm{H}_{2} \mathrm{O}_{2}(25 \mu \mathrm{M})$ stimulation or the vehicle 
control. C, Quantitative PCR for SAMHD1 mRNA in HCT116 cells with or without $\mathrm{H}_{2} \mathrm{O}_{2}(25 \mu \mathrm{M})$ treatment for $2 \mathrm{~h}$. D, Western blot analysis of SAMHD1 in HCT116 cells with or without cisplatin $(5 \mu \mathrm{M})$ treatment. E, Quantitative PCR for SAMHD1 mRNA in HCT116 cells with or without cisplatin $(5 \mu \mathrm{M})$ treatment for $1 \mathrm{~h}$. F, Western blot analysis of SAMHD1 in HCT116 cells with or without doxorubicin $(5 \mu \mathrm{M})$ treatment. G, Quantitative PCR for SAMHD1 mRNA in HCT116 cells with or without doxorubicin $(5 \mu \mathrm{M})$ treatment for $1 \mathrm{~h} . \mathbf{H}$ and I, The lysates of HCT116 cells in with or without $1 \mathrm{~h}$ of cisplatin $(5 \mu \mathrm{M})$ or doxorubicin $(5 \mu \mathrm{M})$ stimulation were immunoprecipitated with the IgG control or anti-SAMHD1 antibody, followed by immunoblotting with the ubiquitin antibody. $\mathbf{J}$ and $\mathbf{K}$, The lysates of HCT116 cells with or without 1 h stimulation with cisplatin $(5 \mu \mathrm{M}, \mathrm{J})$ or doxorubicin $(5 \mu \mathrm{M}, \mathrm{K})$ were immunoprecipitated with the IgG control or anti-SAMHD1 antibody, followed by immunoblotting with the SAMHD1 and CtIP antibodies.

\section{Figure S3. USP7 binds to SAMHD1 and stabilizes its protein expression.}

A, The lysates of Hela cells were immunoprecipitated with the IgG control or anti-SAMHD1 antibody followed by immunoblotting with the USP7 and SAMHD1 antibodies. B, Flag-tagged USP7 plasmid was transfected into Hela cells as indicated. Total lysates were subjected to IP and Western blot with the anti-Flag antibody. C, The lysates of HEK293 cells were immunoprecipitated with the IgG control or anti-SAMHD1 antibody followed by immunoblotting with the USP7 and SAMHD1 antibodies. D, Flag-tagged HEK293 plasmid was transfected into Hela cells as indicated. Total lysates were subjected to IP and Western blot with the anti-Flag antibody. E, SAMHD1 was colocalized with USP7 in HEK293 cells by immunofluorescence. Scale bar, $10 \mu \mathrm{m}$. F, Western blot analysis of SAMHD1 in H1299 cells without or with P5091treatment at different concentrations for $24 \mathrm{~h}$. G, Western blot analysis of SAMHD1 in HCT116 cells without or with $20 \mu \mathrm{M}$ of P5091 treatment for different durations.

Figure S4. USP7 deubiquitiantes SAMHD1 at K421. 
A, The lysates of HEK293 cells transfected with or without Flag-USP7 transfection for $48 \mathrm{~h}$ were immunoprecipitated with the $\mathrm{IgG}$ control or anti-SAMHD1 antibody followed by immunoblotting with the ubiquitin antibody. B-D, The lysates of SW480

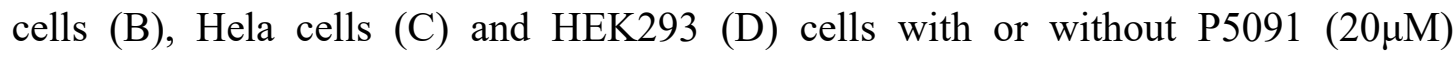
pretreatment for $24 \mathrm{~h}$ were immunoprecipitated with the IgG control or anti-SAMHD1 antibody followed by immunoblotting with the ubiquitin antibody. E, The lysates of HCT116 cells transfected with Flag-SAMHD1 WT/Flag-SAMHD1 K421R/Flag-SAMHD1 K492R/Flag-SAMHD1 K494R, HA-Ub for 48h were immunoprecipitated with the Flag antibody followed by immunoblotting with the HA antibody. F, Sequence alignment of SAMHD1 homologs surrounding K421 in various species. The letter $\mathrm{K}$ in red indicates the lysine residues at 421 of SAMHD1. G-I, Mass spectrogram of ubiquitination sites of K421, K492 and K494.

Figure S5. SAMHD1 promotes cell survival and reduces apoptosis under genotoxic insults

A-B, SW480 shNC and shSAMHD1 cells or shSAMHD1-Flag and shSAMHD1-Flag-SAMHD1 cells were treated with cisplatin at different concentrations for $24 \mathrm{~h}$. Cell viability was assessed by CCK8 assay. C-F, HCT116 or SW480 shNC and shSAMHD1 cells or shSAMHD1-Flag and shSAMHD1-Flag-SAMHD1 cells were treated with cisplatin $(20 \mu \mathrm{M})$ for different durations. Cell viability was assessed by CCK8 assay. G-H, SW480 shNC and shSAMHD1 cells or shSAMHD1-Flag and shSAMHD1-Flag-SAMHD1 cells were treated with cisplatin $(20 \mu \mathrm{M})$ for $24 \mathrm{~h}$ followed by PI and FITC-Annexin V staining and FACS analysis. Bar graph represents percentage of apoptotic cells from three independent experiments. H, HCT116 cells with or without 4 h of cisplatin $(5 \mu \mathrm{M})$ stimulation were stained with the anti-CtIP and anti- $\gamma \mathrm{H} 2 \mathrm{AX}$ antibodies, DAPI, respectively, for immunofluorescence analysis. Scale bar, $10 \mu \mathrm{m}$. Data are expressed as mean \pm SEM. $* \mathrm{P}<0.05 ; * * \mathrm{P}<0.01 ; * * * \mathrm{P}<0.001$ 


\section{genotoxic insults}

A, The lysates of HCT116 cells transfected with Flag-USP7 with or without cisplatin $(5 \mu \mathrm{M})$ stimulation for $0.5 \mathrm{~h}$ or $1 \mathrm{~h}$ were immunoprecipitated with anti-Flag antibody followed by immunoblotting with the SAMHD1 and Flag antibodies. B, The lysates of HCT116 cells transfected with Flag-SAMHD1 in with or without cisplatin $(5 \mu \mathrm{M})$ stimulation for $0.5 \mathrm{~h}$ or $1 \mathrm{~h}$ were immunoprecipitated with the anti-Flag antibody followed by immunoblotting with USP7 and Flag antibodies. C, The lysates of HCT116 cells with or without doxorubicin $(5 \mu \mathrm{M})$ stimulation for $1 \mathrm{~h}$ were immunoprecipitated with the IgG control or anti-SAMHD1 antibody followed by immunoblotting with the USP7 and SAMHD1 antibodies. D, Western blot analysis of SAMHD1 in HCT116 cells pretreated with or without P5091 $(20 \mu \mathrm{M})$ followed by stimulation with doxorubicin $(5 \mu \mathrm{M})$ and the vehicle control for 1 h. E and F, HCT116 shNC and shUSP7 cells or shUSP7-Flag and shUSP7-Flag-SAMHD1 cells were treated with doxorubicin at different concentrations for $24 \mathrm{~h}$. Cell viability was assessed by CCK8 assay. G and H, HCT116 shNC and shUSP7 cells or shUSP7-Flag and shUSP7-Flag-SAMHD1 cells were treated with doxorubicin $(10 \mu \mathrm{M})$ for $24 \mathrm{~h}$ followed by 7-AAD and Annexin V-APC staining and FACS analysis. I, HCT116 cells treated with doxorubicin $(10 \mu \mathrm{M})$ for $24 \mathrm{~h}$ in the presence or absence of P5091 $(20 \mu \mathrm{M})$ administration followed by with7-AAD and Annexin V-APC staining and FACS analysis. Bar graph represents percentage of apoptotic cells from three independent experiments. Data are expressed as mean $\pm \mathrm{SEM}$. $* \mathrm{P}<0.05 ; * * \mathrm{P}<0.01$; $* * * \mathrm{P}<0.001$.

Figure S7. USP7 is highly expressed in carcinomas of various origins and positively correlates with SAMHD1.

A-C, Semiquantitative immunohistochemical analysis of USP7 expression in colonic adenocarcinoma $(A, n=30)$, lung adenocarcinoma $(B, n=48)$, thyroid carcinoma $(C$, $\mathrm{n}=58$ ) and their paired peritumoral tissues. $* * * \mathrm{P}<0.001$ (Mann-Whitney test). D-K, Correlation between USP7 and SAMHD1 expressions in various malignant human neoplasms based on the GEPIA database. 
A

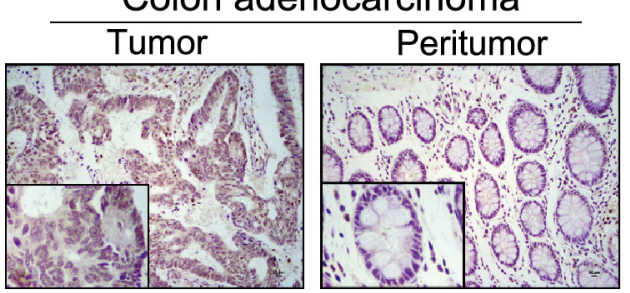

C

E

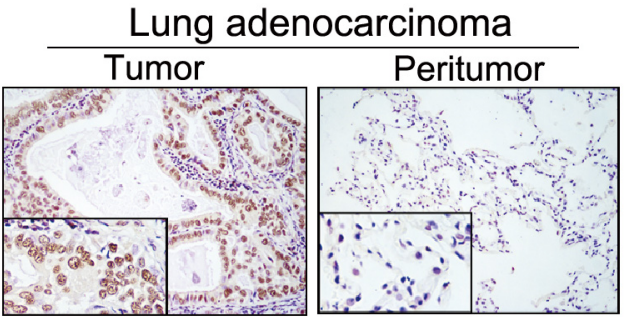

Thyroid carcinoma

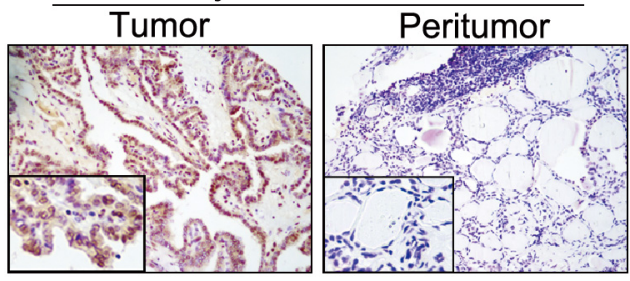

G

$\mathrm{H}$

$\mathrm{H}_{2} \mathrm{O}_{2}$ Oh $15 \mathrm{~m} 30 \mathrm{~m} \quad 1 \mathrm{~h}$

SAMHD1

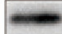

$\beta$-actin

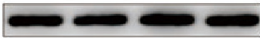

$\beta$-actin
B

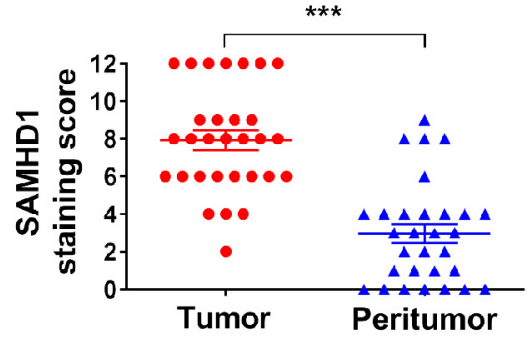

D

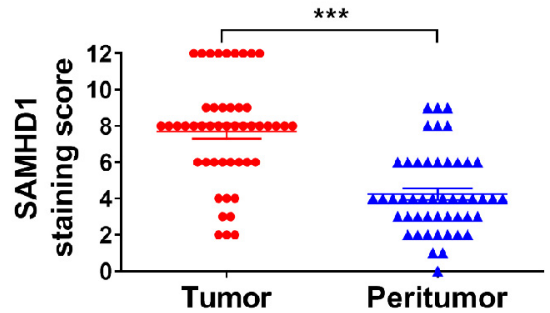

F

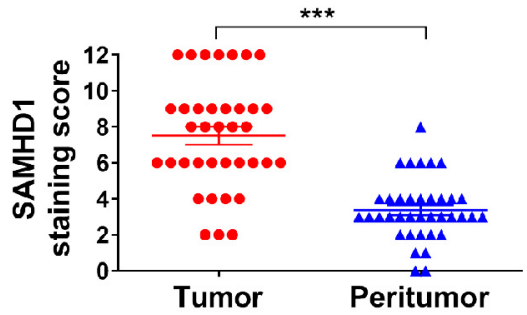

$\mathrm{H}_{2} \mathrm{O}_{2}$

IP IgG SAMHD1

Ub

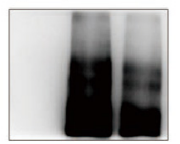

흘 $\begin{gathered}\text { SAMHD1 } \\ \beta \text {-actin }\end{gathered}$

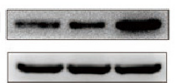

$\mathrm{K}$

SAMHD1 $\mathrm{yH} 2 A X$

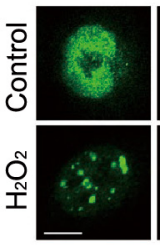

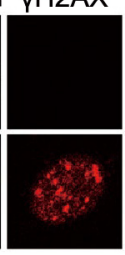

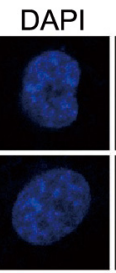

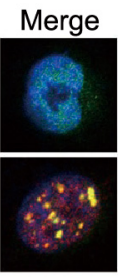

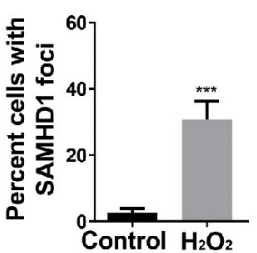

L

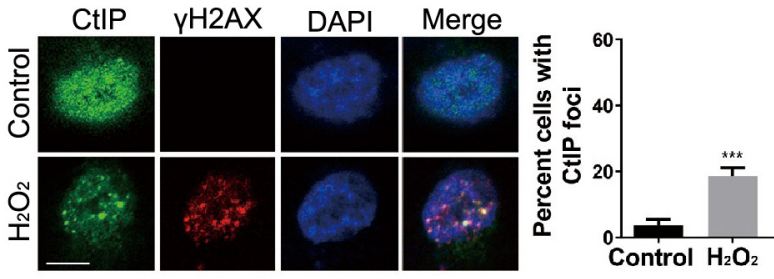


Figure 2

A

\begin{tabular}{ccc}
\hline Accession & Description & Sum Score \\
\hline Q13200 & PSMD2 & 3.470 \\
Q1KMD3 & HNRNPUL2 & 3.417 \\
Q93009 & USP7 & 3.364 \\
Q13895 & Bystin & 3.283 \\
Q16531 & DDB1 & 3.282 \\
P62195 & PSMC5 & 3.269 \\
P29692 & EEF1D & 3.208 \\
Q9BZH6 & FBXW11 & 3.172 \\
Q9UG63 & ABCF2 & 3.002 \\
\hline
\end{tabular}

B

C

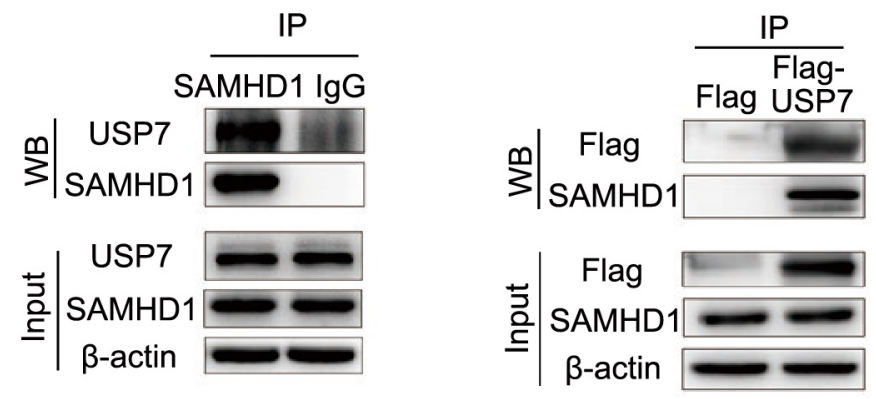

$\mathrm{D}$

E

$\mathrm{F}$

GST-

Input GST SAMHD1

Flag-USP7

Flag- Input GST USP7 SAMHD1 UST-

GSTSAMHD1

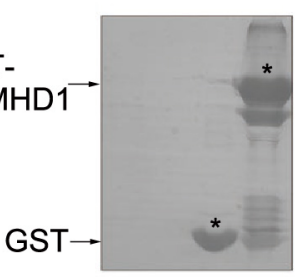

WT

\begin{tabular}{|l|l|l|}
\hline 114 & & \\
\hline NtD & SAM \\
\hline
\end{tabular} HD

583

$\triangle \mathrm{CtD} \quad \mathrm{NtD}|\mathrm{SAM}| \mathrm{HD}$

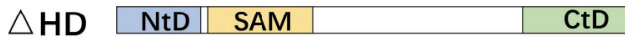

\begin{tabular}{l|l|l||l|}
\hline$\triangle S A M$ & NtD & HD & CtD \\
\hline
\end{tabular}

\begin{tabular}{l|l|l||l|}
\hline$N$ NtD & SAM & HD & CtD \\
\hline
\end{tabular}
G

Flag-USP7

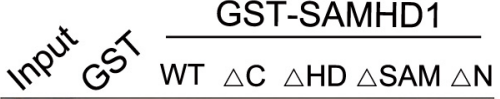

GST-

SAMHD1 $\rightarrow$
GST $\rightarrow$

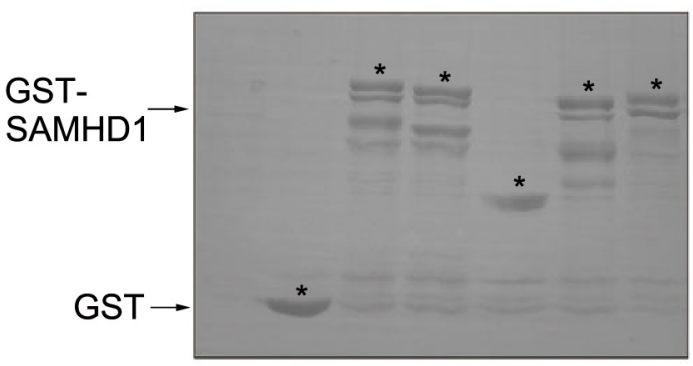

$\mathrm{H}$

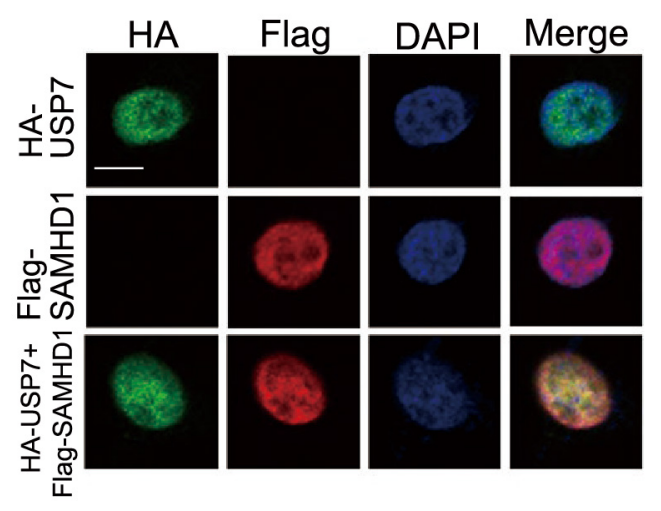


Figure 3

A

Flag-USP7 $-++\quad+$

Flag

SAMHD1

$\beta$-actin

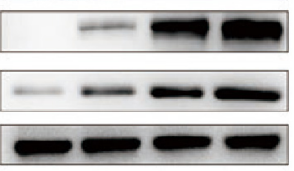

B

USP7 siRNA - \#1 \#2 \#3

USP7
SAMHD1
$\beta$-actin

C
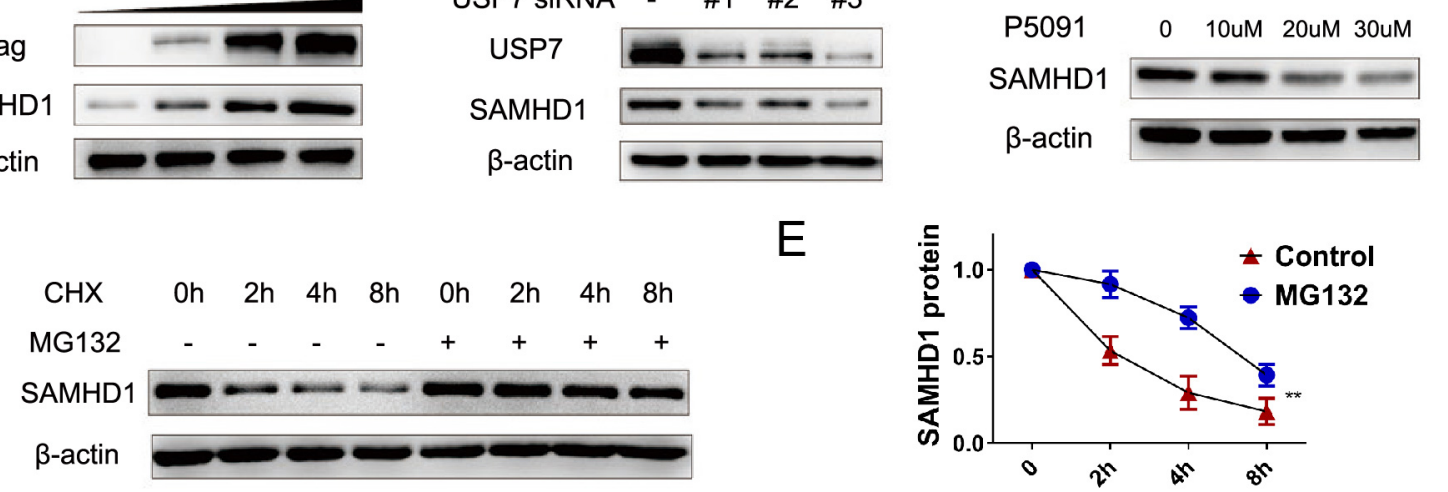

E

D

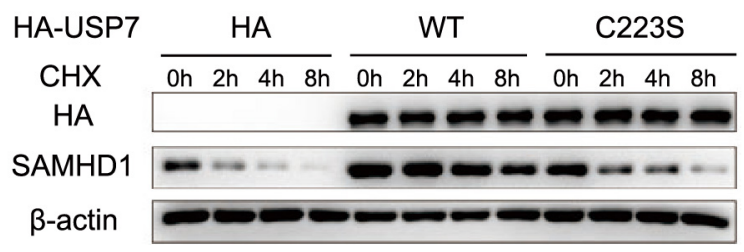

$\mathrm{H}$
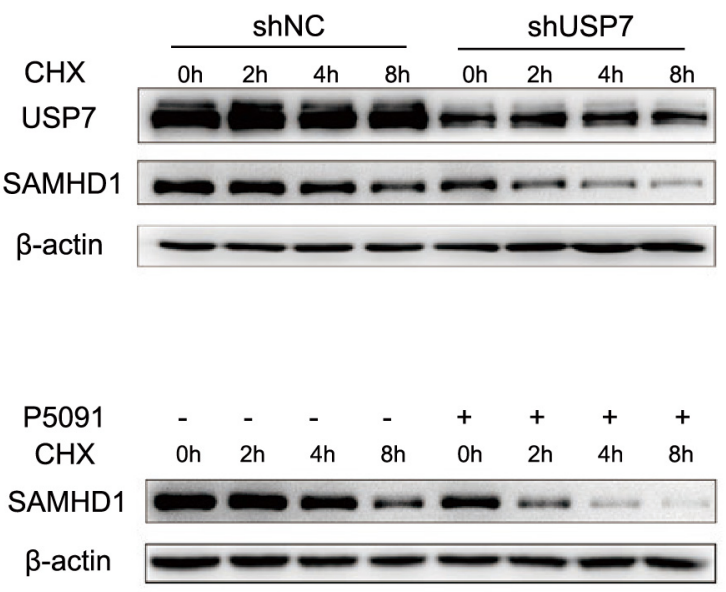

G
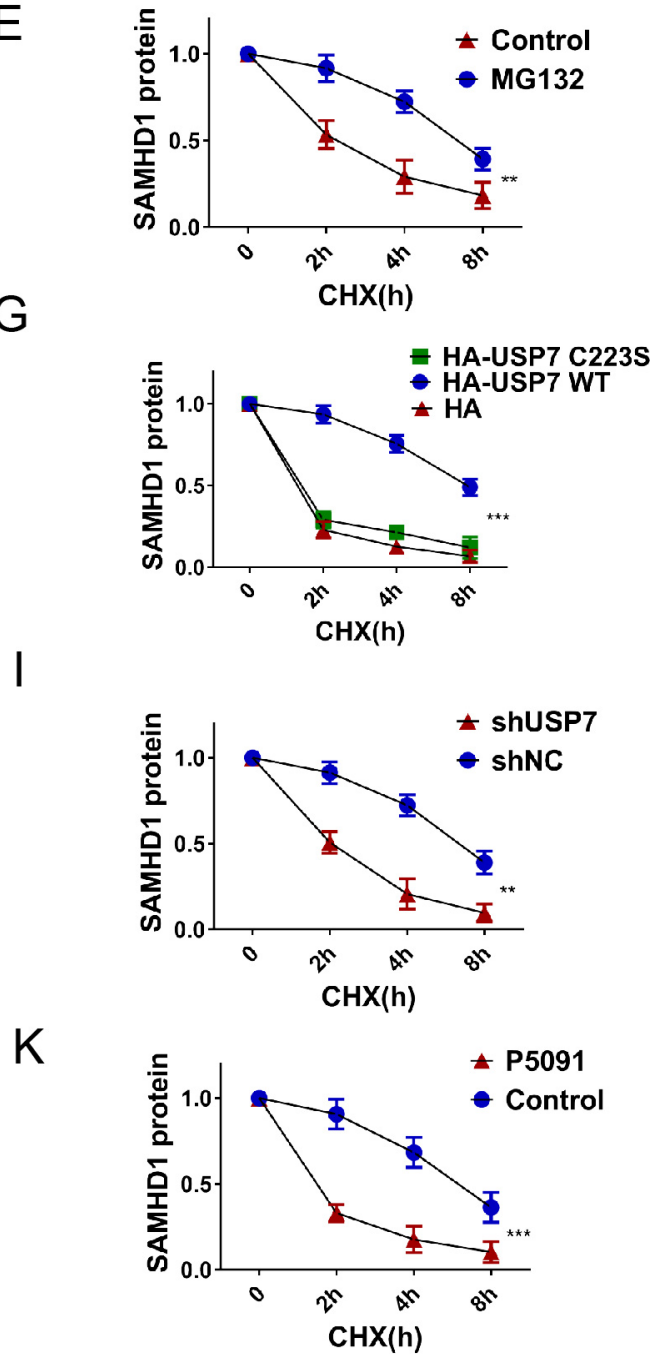
Figure 4

A Flag-USP7 + - +

IP IgG SAMHD1

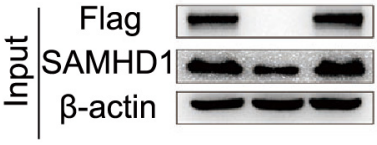

D

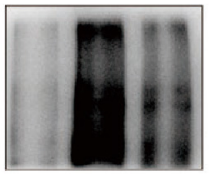

\section{Myc-USP7} Flag-SAMHD1

HA-Ub

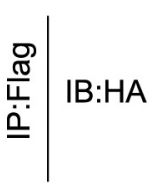

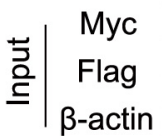

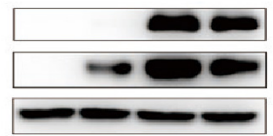

G

$\begin{array}{llllll}\text { Myc-USP7 } & - & + & - & + & - \\ \text { HA-Ub } & + & + & + & + & -\end{array}$
Flag-SAMHD1 WT K494R -
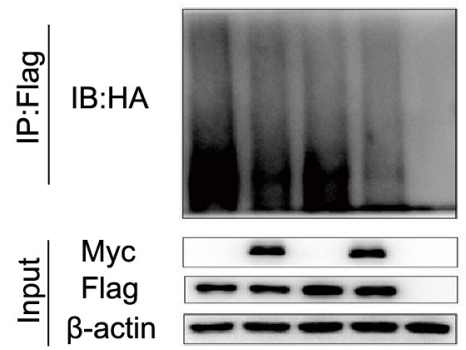

B
ShUSP7 - - +

IP IgG SAMHD1

E

Myc-USP7

HA-Ub

Flag-SAMHD1

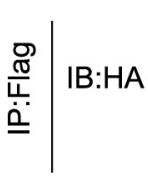

흘 $\begin{gathered}\text { Myc } \\ \text { Flag } \\ \beta \text {-actin }\end{gathered}$

$\mathrm{H}$

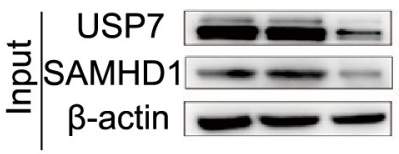

C

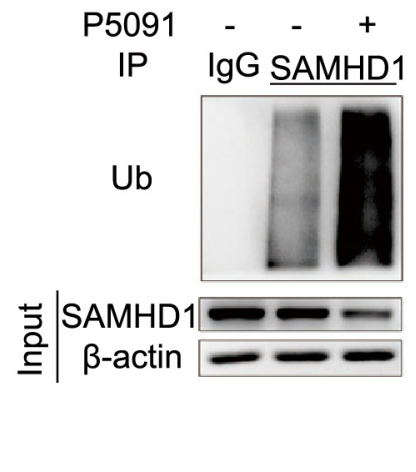

HA-Ub Myc-USP7 Flag-SAMHD1
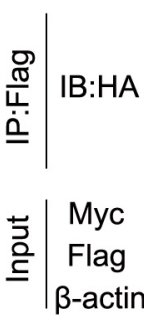
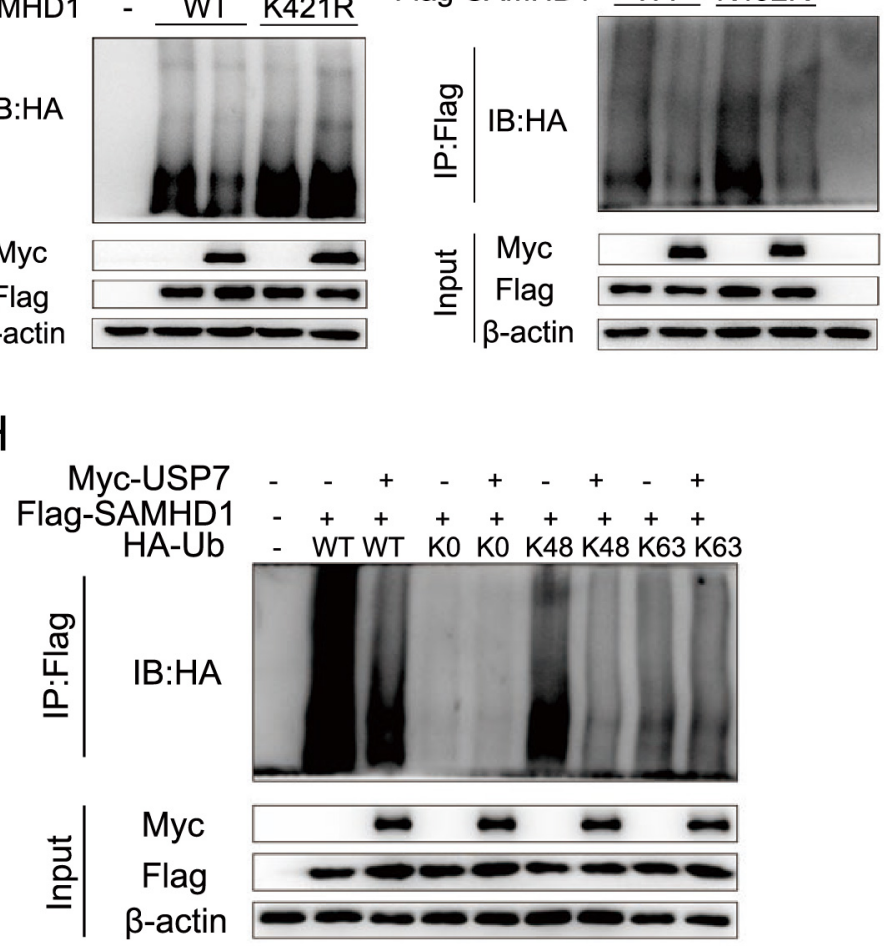
A

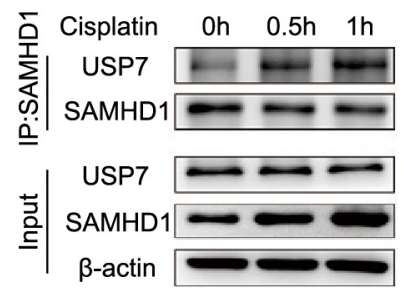

B
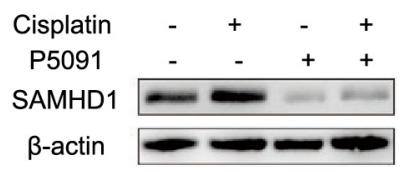

C

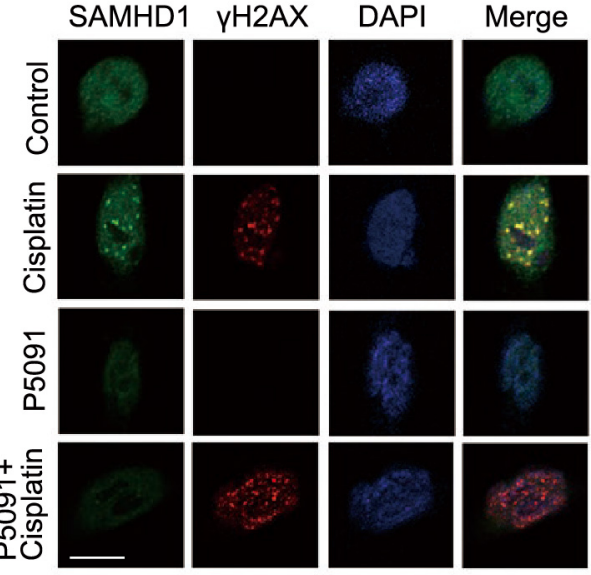

E
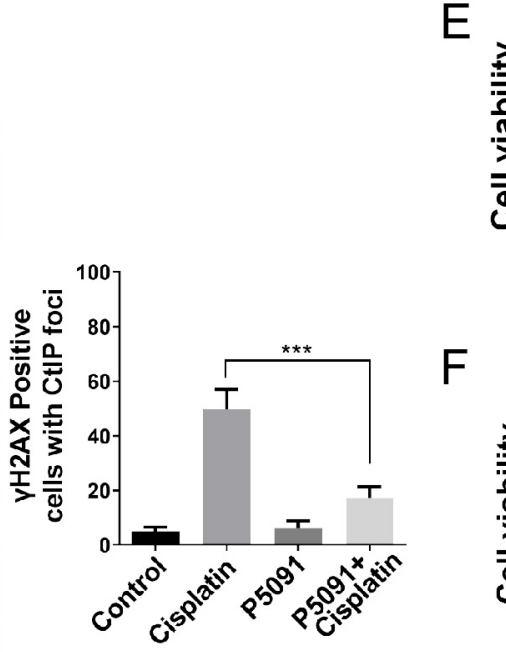

$\mathrm{H}$

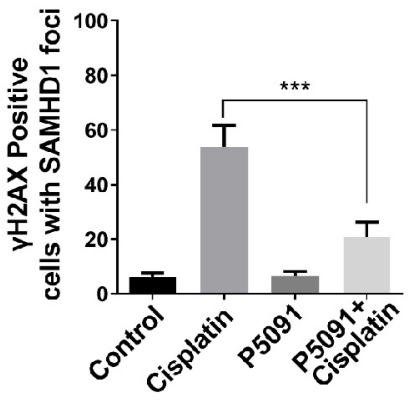

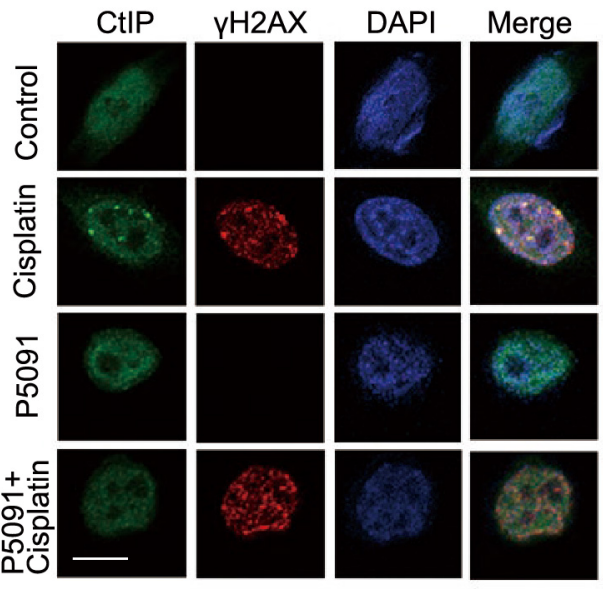

Untreated Cisplatin
1220\%

$\mathrm{PI}$
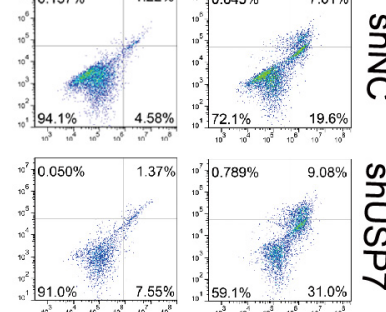

FITC-AnnexinV

4 Untreated Cisplatin

PI

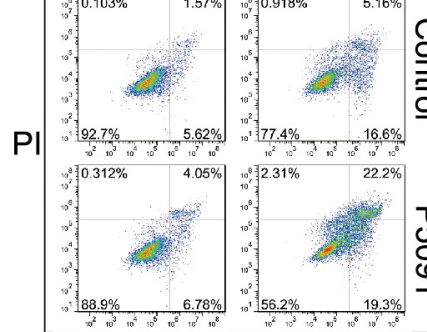

FITC-AnnexinV
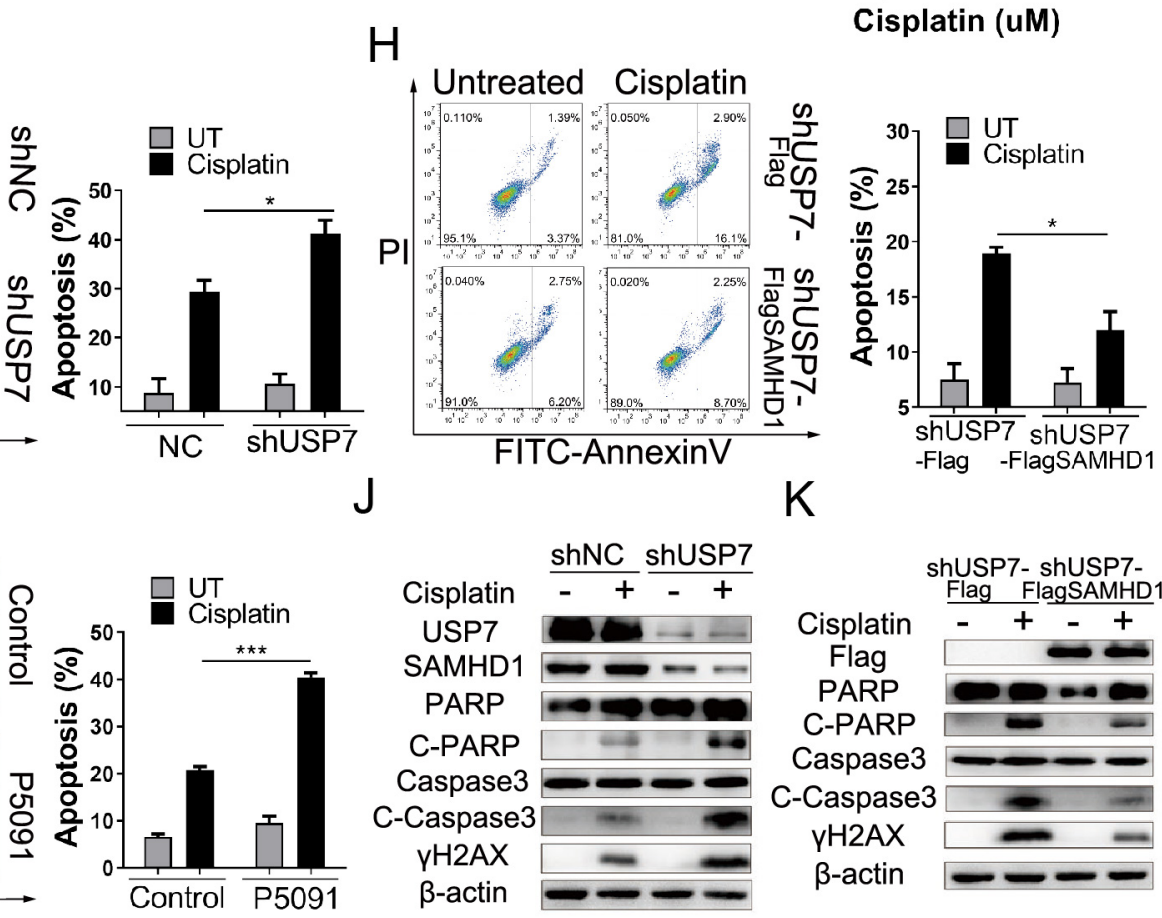

shUSP7- shUSP7Flag FlagSAMHD1

Cisplatin Flag PARP C-PARP Caspase3 C-Caspase 3 YH $2 A X$ $\beta$-actin 
A

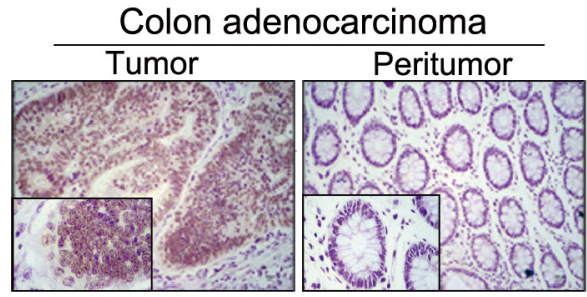

C

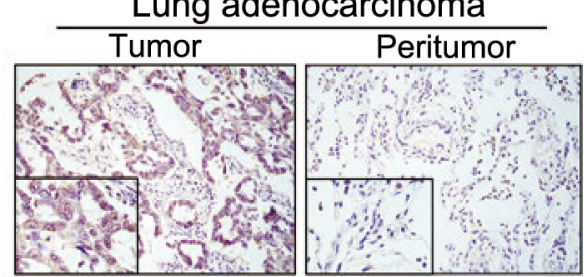

E
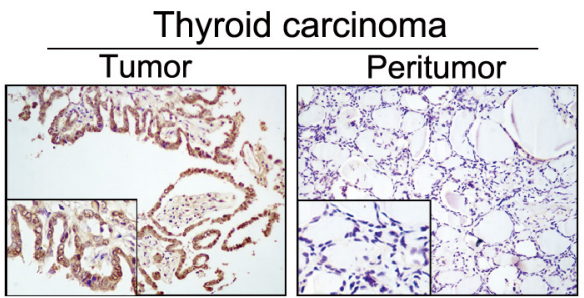

$\mathrm{G}$ Colon adenocarcinoma

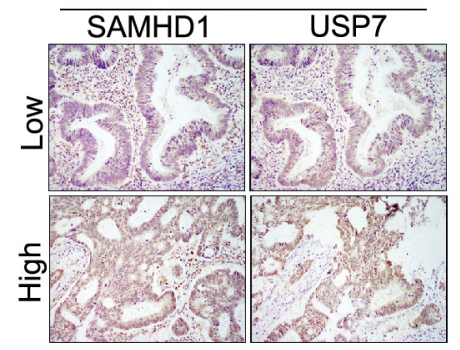

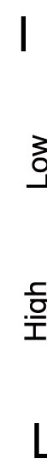

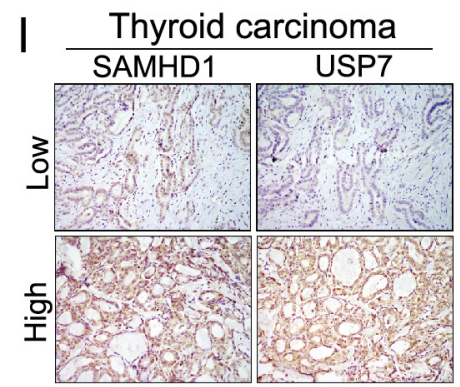

$\mathrm{L}$

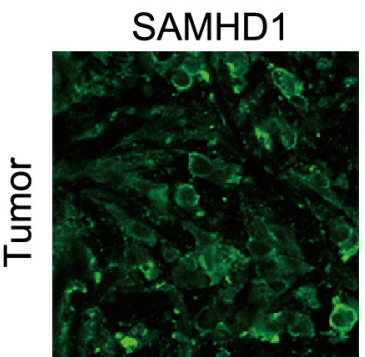

ํㅡㅁ

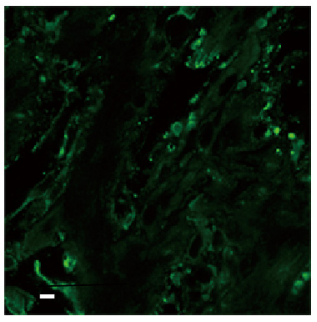

Correlation $\mathrm{P}<0.001 \mathrm{r}=0.603$

USP7
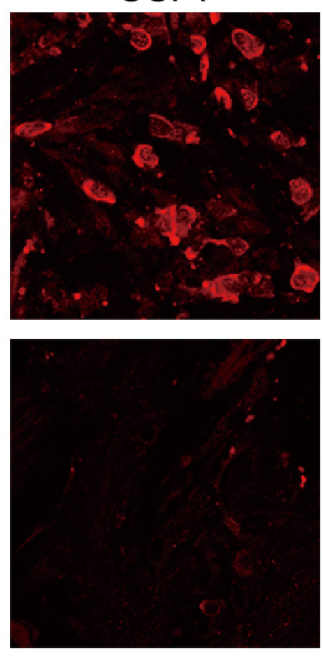

B

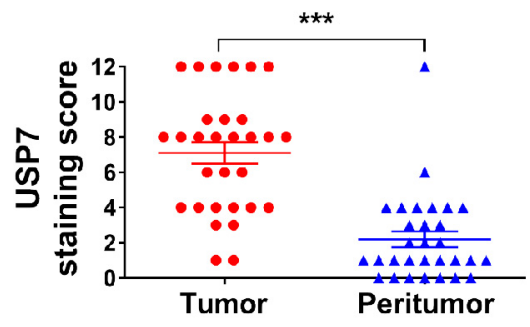

D

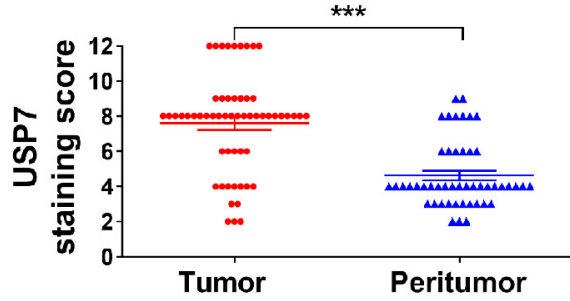

F

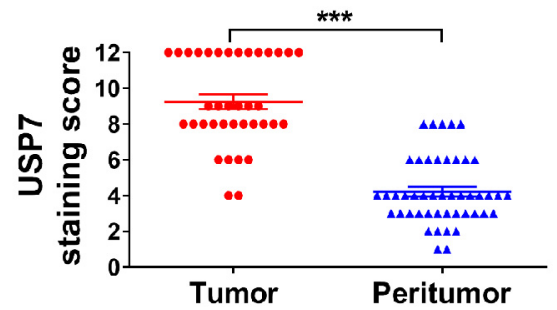

$\mathrm{H}$
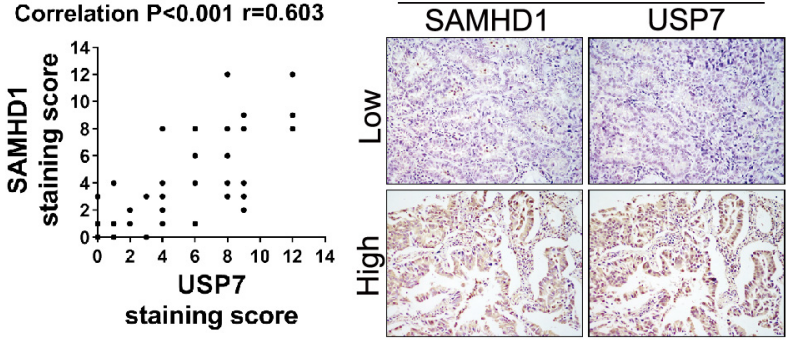

Correlation $\mathrm{P}<0.001 \quad r=0.491$

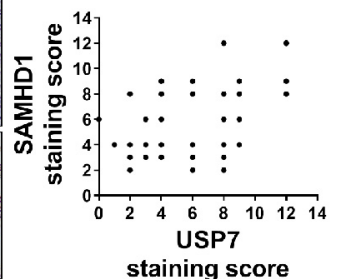

$\mathrm{J}$
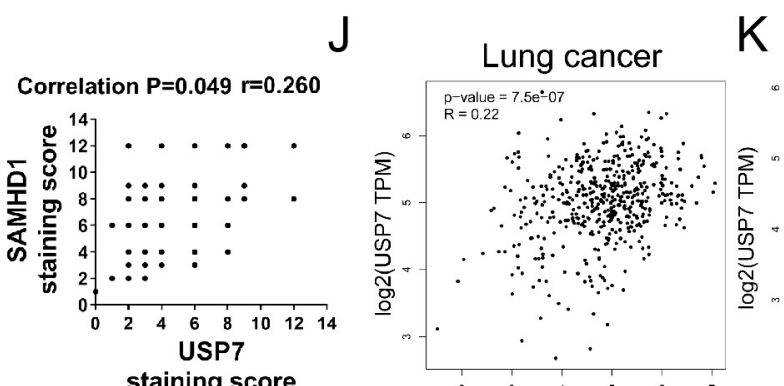

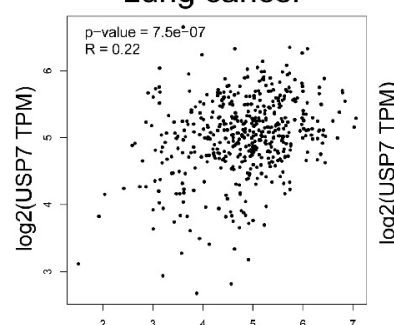

$\log 2$ (SAMHD1 TPM)

DAPI

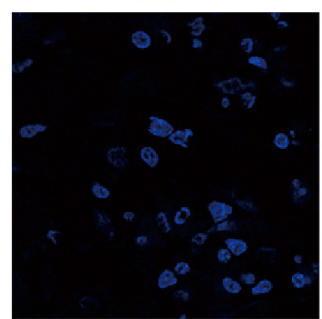

Merge
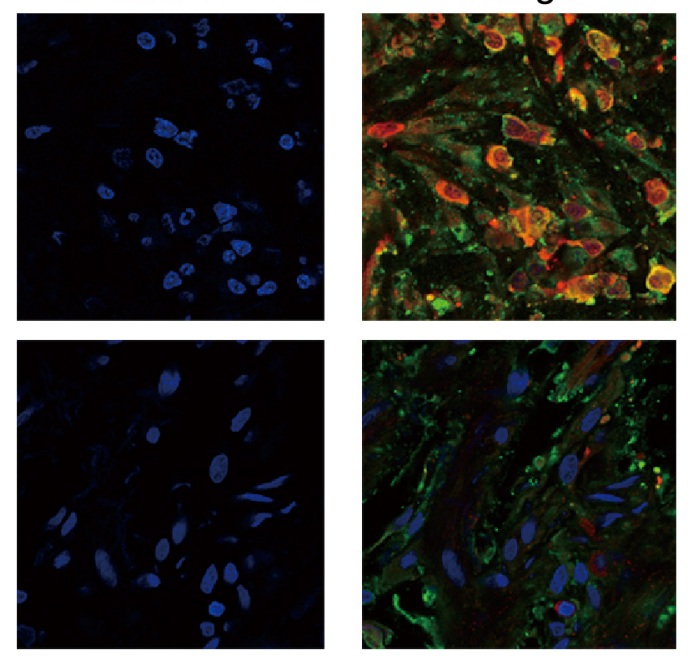

Thyroid cancer

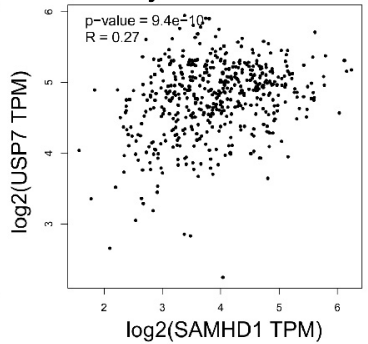

$\log 2($ SAMHD1 TPM) 
Figure S1

A

\section{SAMHD1 expression}

\begin{tabular}{lllllll}
\cline { 2 - 3 } Colon adenocarcinoma & Low & Medium & High & $\mathrm{n}$ &
\end{tabular}

\begin{tabular}{lccccc} 
Tumor & 4 & 15 & 11 & 30 & $<0.001$ \\
Peritumor & 25 & 4 & 1 & 30 & \\
\hline
\end{tabular}

B

\section{SAMHD1 expression}

\begin{tabular}{cccccc} 
Lung adenocarcinoma & Low & Medium & High & $\mathrm{n}$ & $\mathrm{P}$ \\
\hline Tumor & 8 & 25 & 15 & 48 & $<0.001$ \\
Peritumor & 33 & 12 & 3 & 48 & \\
\hline
\end{tabular}

\section{SAMHD1 expression}

$\begin{array}{cccccc}\text { Thyroid carcinoma } & \text { Low } & \text { Medium } & \text { High } & \mathrm{n} & \mathrm{P} \\ \text { Tumor } & 13 & 23 & 22 & 58 & <0.001 \\ \text { Peritumor } & 49 & 9 & 0 & 58 & \\ & & & & & \end{array}$

D

SAMHD1 expression

\begin{tabular}{ccccccc} 
Colon adenocarcinoma & Low & High & $\mathrm{n}$ & HR & $95 \% \mathrm{Cl}$ & $\mathrm{P}$ \\
\hline Live & 78 & 45 & 123 & 2.15 & $1.07-4.32$ & 0.020 \\
Death & 15 & 19 & 34 & & & \\
\hline
\end{tabular}

E

SAMHD1 expression

\begin{tabular}{ccccccc} 
Lung adenocarcinoma & Low & High & $\mathrm{n}$ & $\mathrm{HR}$ & $95 \% \mathrm{Cl}$ & $\mathrm{P}$ \\
\cline { 2 - 6 } Live & 22 & 86 & 108 & 3.34 & $1.65-6.74$ & 0.030
\end{tabular}

$\begin{array}{llll}\text { Death } & 3 & 62 & 65\end{array}$

$\mathrm{F}$

\begin{tabular}{ccccccc} 
& \multicolumn{2}{c}{ SAMHD1 expression } & & & \\
Glioblastoma & Low & High & $\mathrm{n}$ & HR & $95 \% \mathrm{Cl}$ & $\mathrm{P}$ \\
\hline Live & 4 & 23 & 27 & 1.99 & $1.17-3.38$ & 0.034 \\
Death & 9 & 87 & 96 & & & \\
\hline
\end{tabular}

\begin{tabular}{ccccccc}
\hline & \multicolumn{9}{l}{ SAMHD1 expression } & & \\
Glioma & Low & High & $\mathrm{n}$ & $\mathrm{HR}$ & $95 \% \mathrm{Cl}$ & $\mathrm{P}$ \\
\hline Live & 142 & 39 & 181 & 1.56 & $1.00-2.43$ & 0.026 \\
Death & 76 & 35 & 111 & & & \\
\hline
\end{tabular}


Figure S3

A

SAMHD1/gG

USP7

3 SAMHD1

\begin{tabular}{c|c} 
USP7 \\
흐을 & SAMHD1 \\
으-actin
\end{tabular}

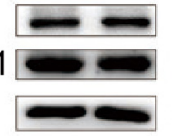

B

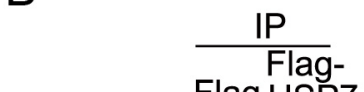

$\stackrel{m}{\text { FAMHD1 }}$

\begin{tabular}{c|c} 
Flag \\
읃 \\
SAMHD1 \\
$\beta$-actin
\end{tabular}

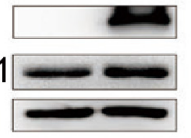

C

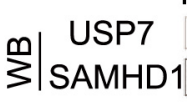

$\frac{\text { IP }}{\operatorname{lgGSAMHD1}}$

D

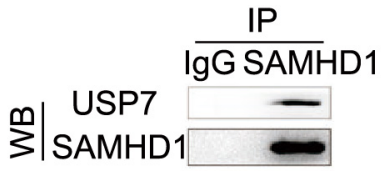

芌| $\begin{gathered}\text { USP7 } \\ \text { SAMHD1 } \\ \text { 으-actin }\end{gathered}$

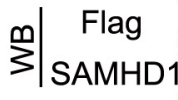

Flag USP7

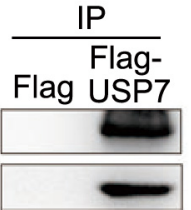

$\stackrel{\text { 흔 }}{\text { SAMHD }} \begin{gathered}\text { Flag } \\ \beta \text {-actin }\end{gathered}$

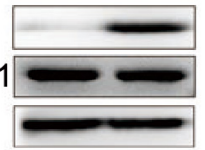

$E$
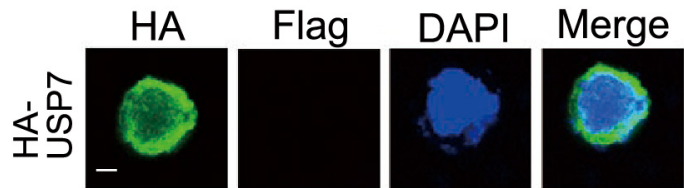

F
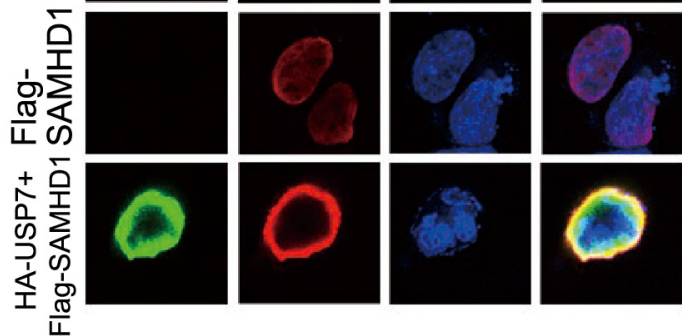

P5091 O 10uM 20uM 30uM SAMHD1

$\beta$-actin

G

P5091 Oh 6h 12h 24h SAMHD1 $-\infty$ $\beta$-actin 
Figure S4

A

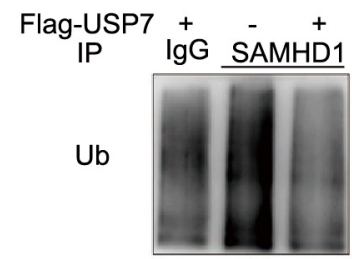

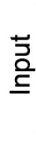

Flag

SAMHD1

$\beta$-actin

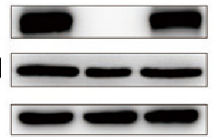

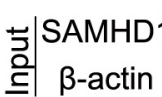

SW480 $\begin{array}{ccc}\text { P5091 } & - & - \\ \text { IP } & +\end{array}$

$\mathrm{Ub}$
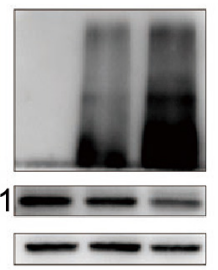

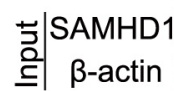

$\mathrm{F}$
C

Hela P5091 - - +

IP IgG SAMHD1

Ub

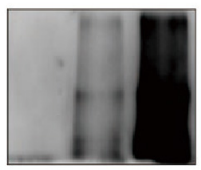

Ub

IP IgG SAMHD1

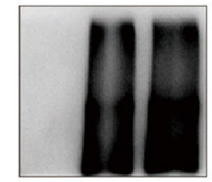

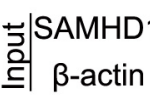

Flag-SAMHD1 WT K421R K492R K494R

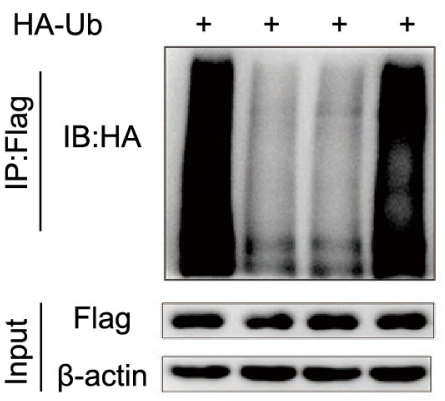

Homo sapiens (415-427) Mus musculus (427-439)

Pan troglodytes (415-427)

Rattus norvegicus (420-432)

Gallus gallus (406-418)

Bos taurus (404-416)
DMEAYTKLTDNIF DMEAFTKLTDNIF DMEAYTKLTDNIF DMEAFSKLTDNIF DMEAYTKLTDNIY DMEAFTKLTDNIF

\section{K421}

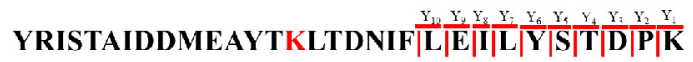

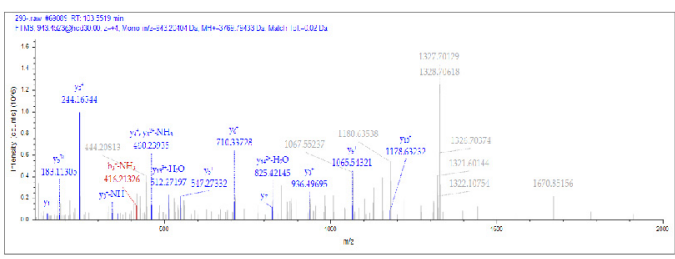

$\mathrm{H}$

K492

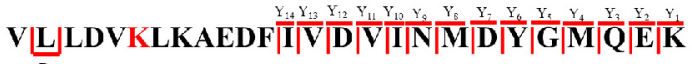

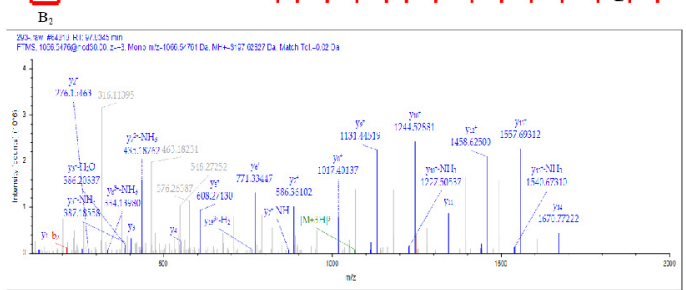

\section{K494}

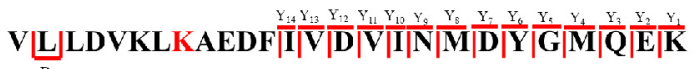

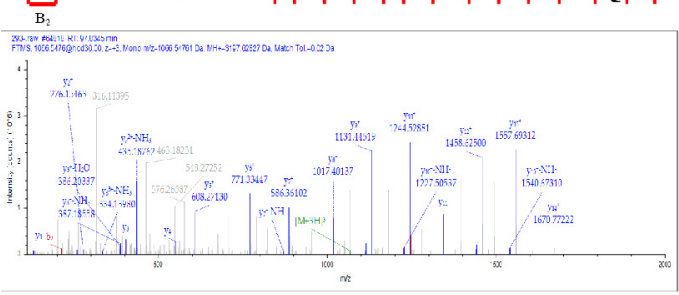


Figure S5

A
B
C

SW480

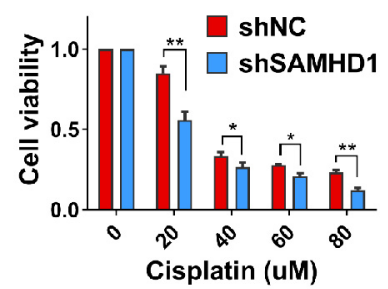

E

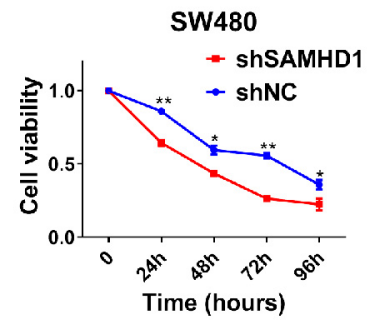

$\mathrm{H}$

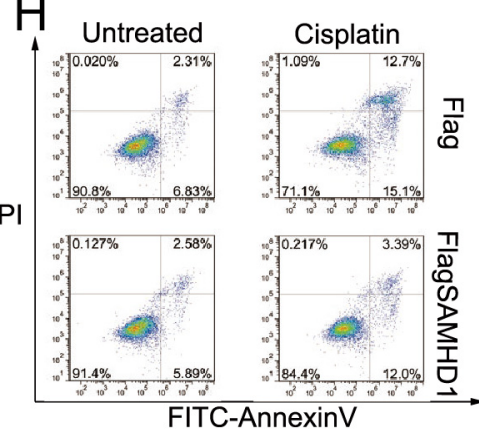

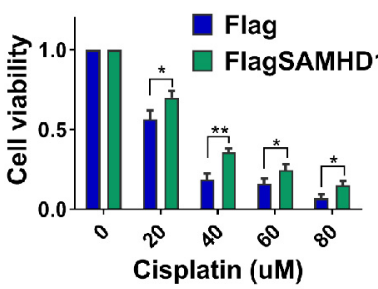

F

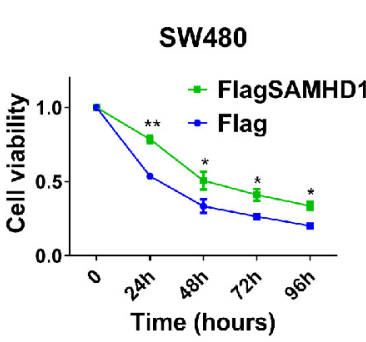

Pl

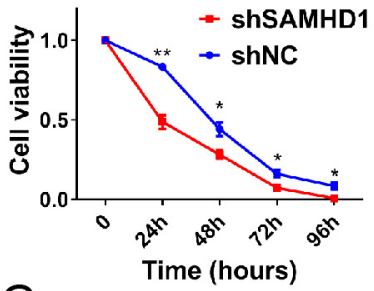

G
HCT116

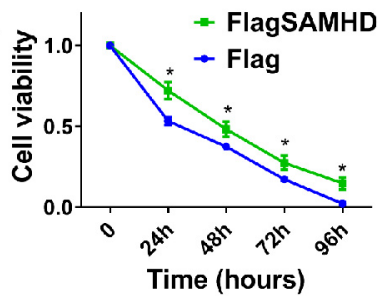

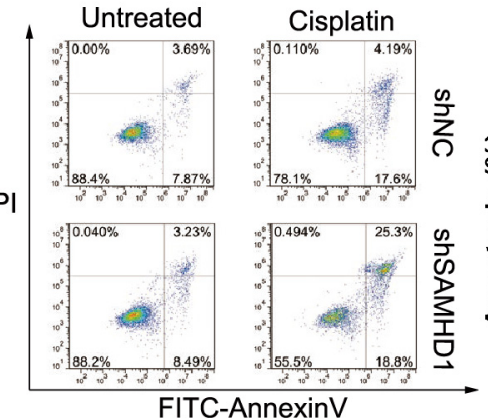

I
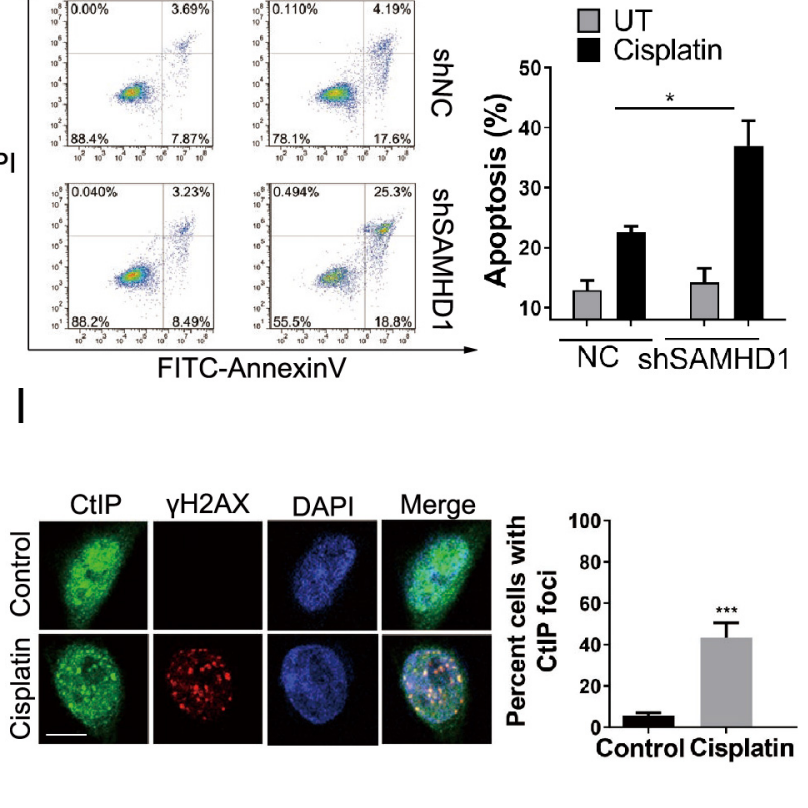


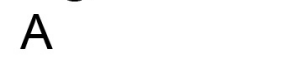

Cisplatin on $0.5 \mathrm{~h} 1 \mathrm{~h}$

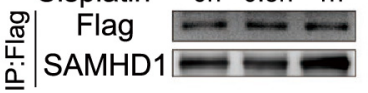

\begin{tabular}{c|c} 
Flag \\
흘 \\
SAMHD1 \\
$\beta$-actin
\end{tabular}

E

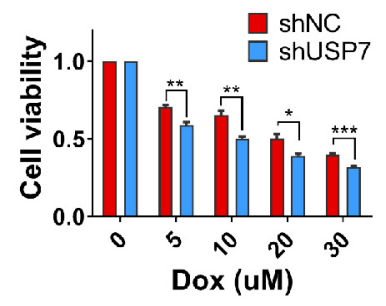

$\mathrm{H}$

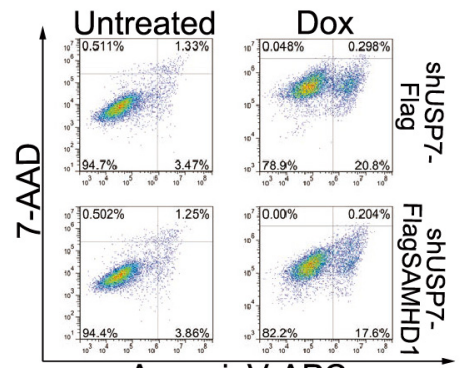

AnnexinV-APC
B

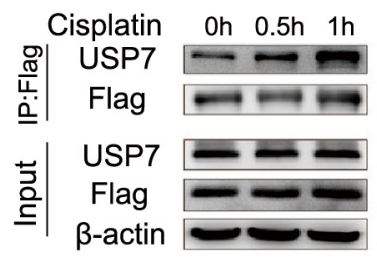

C

Dox

- $\quad+$

IP IgG SAMHD1
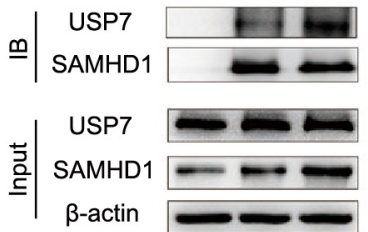

$\begin{array}{ccccc}\text { Dox } & - & + & - & + \\ \text { P5091 } & - & - & + & +\end{array}$

SAMHD1 $-\infty$

$\beta$-actin
F

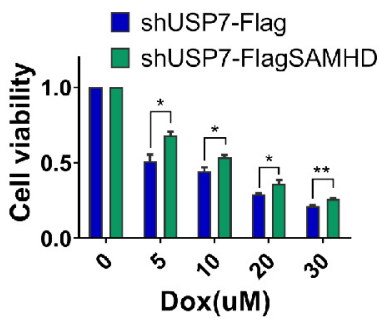

Dox(UM)

\section{G}
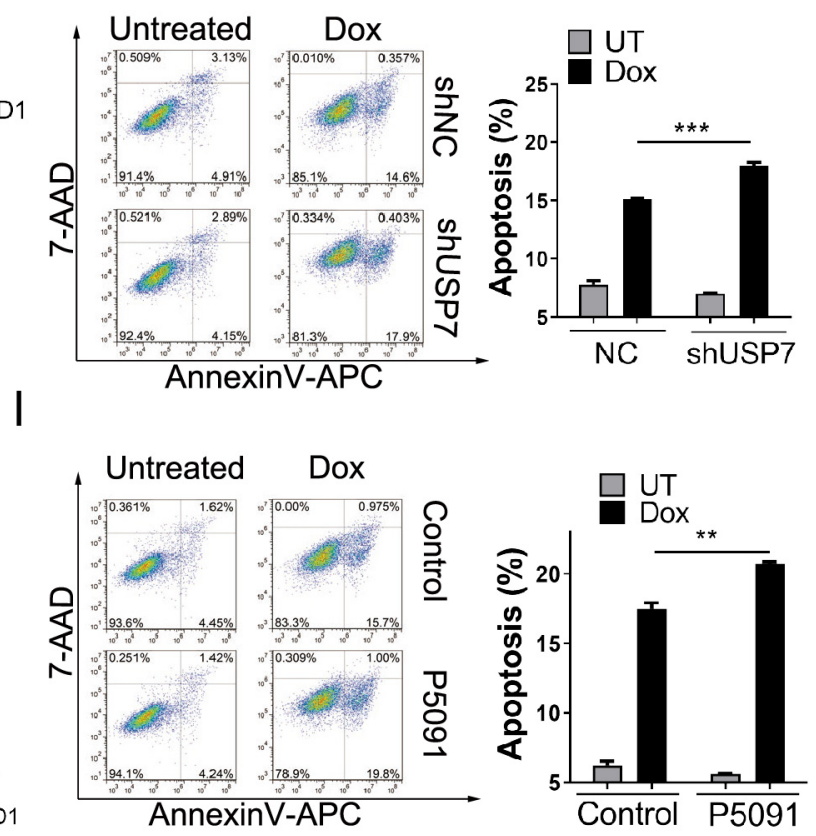
Figure S7

A

\begin{tabular}{cccccc}
\hline & \multicolumn{5}{c}{ USP7 expression } \\
\cline { 2 - 5 } Colon adenocarcinoma & Low & Medium & High & $\mathrm{n}$ & $\mathrm{P}$ \\
\hline Tumor & 10 & 11 & 9 & 30 & $<0.001$ \\
Peritumor & 28 & 1 & 1 & 30 & \\
\hline
\end{tabular}

B

\begin{tabular}{cccccc}
\hline & \multicolumn{5}{c}{ USP7 expression } \\
\cline { 2 - 5 } Lung adenocarcinoma & Low & Medium & High & $\mathrm{n}$ & $\mathrm{P}$ \\
\hline Tumor & 11 & 29 & 8 & 48 & $<0.001$ \\
Peritumor & 34 & 12 & 2 & 48 & \\
\hline
\end{tabular}

C

\begin{tabular}{cccccc}
\hline & \multicolumn{5}{c}{ USP7 expression } \\
\cline { 2 - 5 } Thyroid carcinoma & Low & Medium & High & $\mathrm{n}$ & $\mathrm{P}$ \\
\hline Tumor & 2 & 25 & 31 & 58 & $<0.001$ \\
Peritumor & 38 & 19 & 1 & 58 & \\
\hline
\end{tabular}

D

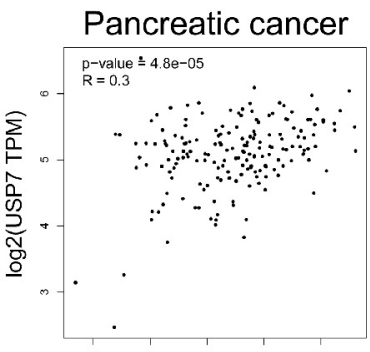

$\log 2$ (SAMHD1 TPM)

$\mathrm{H}$

Gastric cancer

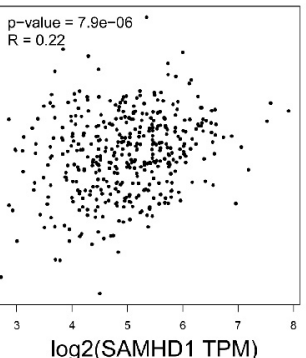

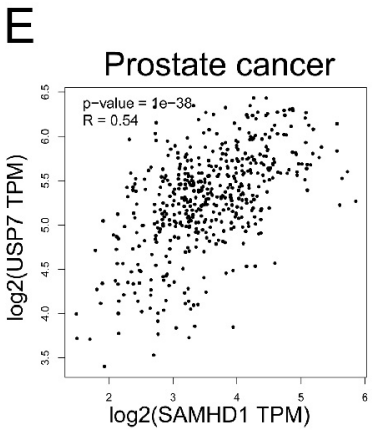

I

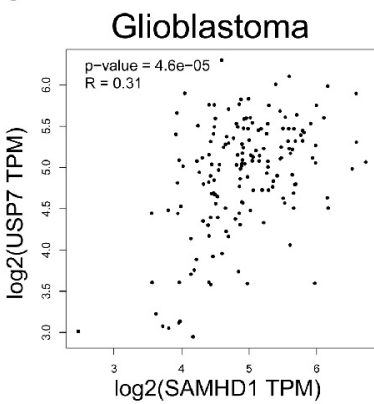

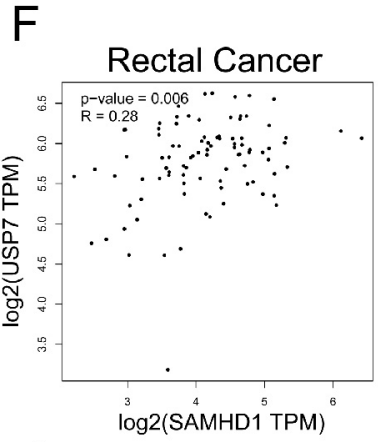

G

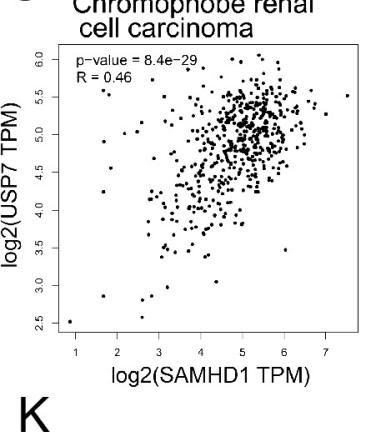

Hepatocellular carcinoma

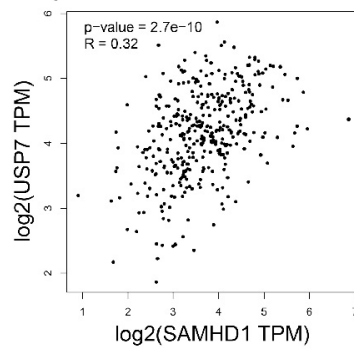

